\title{
A Generalized KdV Equation of Neglecting the Highest-Order Infinitesimal Term and Its Exact Traveling Wave Solutions
}

\author{
Xianbin Wu, ${ }^{1}$ Weiguo Rui, ${ }^{2}$ and Xiaochun Hong ${ }^{3}$ \\ ${ }^{1}$ Junior College, Zhejiang Wanli University, Ningbo 315100, China \\ ${ }^{2}$ College of Mathematics, Honghe University, Mengzi, Yunnan 661100, China \\ ${ }^{3}$ College of Statistics and Mathematics, Yunnan University of Finance and Economics, Kunming, Yunnan 650221, China
}

Correspondence should be addressed to Weiguo Rui; weiguorhhu@yahoo.com.cn

Received 3 November 2012; Accepted 15 January 2013

Academic Editor: Julian López-Gómez

Copyright (C) 2013 Xianbin Wu et al. This is an open access article distributed under the Creative Commons Attribution License, which permits unrestricted use, distribution, and reproduction in any medium, provided the original work is properly cited.

\begin{abstract}
We study a generalized $\mathrm{KdV}$ equation of neglecting the highest order infinitesimal term, which is an important water wave model. Some exact traveling wave solutions such as singular solitary wave solutions, semiloop soliton solutions, dark soliton solutions, dark peakon solutions, dark loop-soliton solutions, broken loop-soliton solutions, broken wave solutions of U-form and C-form, periodic wave solutions of singular type, and broken wave solution of semiparabola form are obtained. By using mathematical software Maple, we show their profiles and discuss their dynamic properties. Investigating these properties, we find that the waveforms of some traveling wave solutions vary with changes of certain parameters.
\end{abstract}

\section{Introduction}

In 1995, based on the physical and asymptotic considerations, Fokas [1] derived the following generalized $\mathrm{KdV}$ equation:

$$
\begin{aligned}
\eta_{t}+\eta_{x} & +\alpha \eta \eta_{x}+\beta \eta_{x x x}+\rho_{1} \alpha^{2} \eta^{2} \eta_{x}+\alpha \beta\left(\rho_{2} \eta \eta_{x x x}+\rho_{3} \eta_{x} \eta_{x x}\right) \\
& +\rho_{4} \alpha^{3} \eta^{3} \eta_{x}+\alpha^{2} \beta\left(\rho_{5} \eta^{2} \eta_{x x x}+\rho_{6} \eta \eta_{x} \eta_{x x}+\rho_{7} \eta_{x}^{3}\right)=0
\end{aligned}
$$

which is an important water wave model, where $\alpha=3 \mathrm{~A} / 2$, $\beta=B / 6, \rho_{1}=-1 / 6, \rho_{2}=5 / 3, \rho_{3}=23 / 6, \rho_{4}=1 / 8$, $\rho_{5}=7 / 18, \rho_{6}=79 / 36, \rho_{7}=45 / 36$. Regarding the $\rho_{1}, \rho_{2}, \rho_{3}, \rho_{4}, \rho_{5}, \rho_{6}, \rho_{7}$ as free parameters and using the $\widetilde{\rho}_{4}$ to replace the $\rho_{4} \alpha^{2}$ (i.e., $\rho_{4} \alpha^{2} \mapsto \widetilde{\rho}_{4}$ ), (1) becomes the following PDE:

$$
\begin{gathered}
u_{t}+u_{x}+\alpha u u_{x}+\beta u_{x x x}+\rho_{1} \alpha^{2} u^{2} u_{x}+\alpha \beta\left(\rho_{2} u u_{x x x}+\rho_{3} u_{x} u_{x x}\right) \\
+\widetilde{\rho}_{4} \alpha u^{3} u_{x}+\alpha^{2} \beta\left(\rho_{5} u^{2} u_{x x x}+\rho_{6} u u_{x} u_{x x}+\rho_{7} u_{x}^{3}\right)=0
\end{gathered}
$$

which is given by Tzirtzilakis et al. in [2]; they called it highorder wave equation of KdV type. Just as Tzirtzilakis [2] said these two equations are both water wave equations of $\mathrm{KdV}$ type, which are more physically and practically meaning-ful. The motion described by the model (1) or (2) is a 2-dimensional, inviscid, incompressible fluid (water) lying above a horizontal fiat bottom located at $\bar{y}=-\bar{h}_{0}\left(\bar{h}_{0}\right.$ is a constant), and letting the air above the water. It turns out that, for such a system if the vorticity is zero initially, it remains zero. The fluids (water) analyzed by Fokas are only irrotational flows. In addition, this system is characterized by two parameters $\alpha=3 A / 2, \beta=B / 6$ with $A=a / \bar{h}_{0}, B=\bar{h}_{0}^{2} / \ell^{2}$, where $a$ and $\ell$ are two typical values of the amplitude and of the wavelength of the waves. The parameters $a, \bar{h}_{0}, \ell$ satisfy the condition $a \ll \bar{h}_{0}<\ell$ because the system is a model of short amplitude and long wavelength. Therefore the parameters $\alpha$ and $\beta$ satisfy the condition $0<\alpha<1,0<\beta<1$.

Assuming that the waves are unidirectional and neglecting terms of $O\left(\alpha^{2}, \alpha^{3}, \alpha \beta\right)$, (1) can be reduced to the classical $\mathrm{KdV}$ equation:

$$
\eta_{t}+\eta_{x}+\alpha \eta \eta_{x}+\beta \eta_{x x x}=0 .
$$

In [1], Fokas assumed that $O(B)$ is less than $O(A)$; this implies $O(\beta)<O(\alpha)$. According to this assumption, we easily know 
that $O\left(\alpha^{2} \beta\right)<O\left(\alpha^{3}\right)$ and $O\left(\alpha^{2} \beta\right)<O(\alpha \beta)$. Neglecting two high-order infinitesimal terms of $O\left(\alpha^{3}, \alpha^{2} \beta\right)$, (1) can be reduced to another high-order wave equation of $\mathrm{KdV}$ type [2-5] as follows:

$$
\begin{array}{r}
\eta_{t}+\eta_{x}+\alpha \eta \eta_{x}+\beta \eta_{x x x}+\rho_{1} \alpha^{2} \eta^{2} \eta_{x} \\
+\alpha \beta\left(\rho_{2} \eta \eta_{x x x}+\rho_{3} \eta_{x} \eta_{x x}\right)=0 .
\end{array}
$$

Equation (4) is a special case of (1) for $\rho_{4}=\rho_{5}=\rho_{6}=\rho_{7}=0$. In [1], it was observed that (4) can be reduced by the local transformation of coordinates

$$
\eta=v-\alpha \rho_{1} v^{2}-\beta\left(3 \rho_{1}+\frac{7}{4} \rho_{2}-\frac{1}{2} \rho_{3}\right) v_{x x}
$$

to a completely integrable PDE as follows:

$$
\begin{aligned}
v_{t}- & \frac{3}{2} \beta \rho_{2} v_{x x t}+\beta\left(1-\frac{3}{2} \rho_{2}\right) v_{x x x}+\alpha v v_{x} \\
- & \frac{1}{2} \alpha \beta \rho_{2}\left(v v_{x x x}+2 v_{x} v_{x x}\right) .
\end{aligned}
$$

Equation (6) was first derived in [6] by using the method of bi-Hamiltonian systems, which based on the physical considerations, its Lax Pair was also given in [7].

If only neglecting the highest order infinitesimal term of $O\left(\alpha^{2} \beta\right)$, then (1) can be reduced to a new generalized $\mathrm{KdV}$ equation as follows:

$$
\begin{aligned}
\eta_{t}+ & \eta_{x}+\alpha \eta \eta_{x}+\beta \eta_{x x x}+\rho_{1} \alpha^{2} \eta^{2} \eta_{x} \\
& +\alpha \beta\left(\rho_{2} \eta \eta_{x x x}+\rho_{3} \eta_{x} \eta_{x x}\right)+\rho_{4} \alpha^{3} \eta^{3} \eta_{x}=0 .
\end{aligned}
$$

We call it a generalized KdV equation of neglecting the highest order infinitesimal term. In fact, (7) is another special case of (1) for $\rho_{5}=\rho_{6}=\rho_{7}=0$; it is also third-order approximate equation of KdV type. Of course, on describing dynamical behaviors of water waves, (4) is only a rough approximative model of (1) compared with (7); that is, the precision of model (7) is better than that of model (4) on describing dynamical behaviors of water waves. In other words, model (7) exhibits much richer phenomenology than model (4). Therefore, the investigation of exact traveling wave solutions for (7) are more practically meaningful than that of (4).

On the other hand, under the local transformation

$$
\begin{aligned}
\eta= & +\lambda_{1} \alpha u^{2}+\lambda_{2} \beta u_{x x}+\lambda_{3} \alpha^{2} u^{3} \\
& +\alpha \beta\left(\lambda_{4} u u_{x x}+\lambda_{5} u_{x}^{2}\right),
\end{aligned}
$$

(1) can be reduced to the following generalized Gardner equation [1]:

$$
\begin{aligned}
u_{t}+u_{x} & -\frac{3}{2} \rho_{2} \beta u_{x x t}+\left(1-\frac{3}{2} \rho_{2}\right) \beta u_{x x x}+\alpha u u_{x} \\
& -\frac{1}{2} \rho_{2} \alpha \beta\left(u u_{x x x}+2 u_{x} u_{x x}\right)+3 \mu \alpha^{2} u^{2} u_{x} \\
& -\frac{3}{2} \mu \rho_{2} \alpha^{2} \beta\left(u^{2} u_{x x x}+u_{x}^{3}+4 u u_{x} u_{x x}\right) \\
& +\frac{9}{4} \mu \rho_{2}^{2} \alpha^{2} \beta^{2}\left(u_{x}^{2} u_{x x x}+2 u_{x} u_{x x}^{2}\right)=0,
\end{aligned}
$$

where $\lambda_{i}(i=1,2,3,4,5)$ are certain expressions of $\rho_{i}$. Clearly, (9) is equivalent to the following generalization form of the modified $\mathrm{KdV}$ equation $[1,8]$ under the transformation $-(3 / 2) \rho_{2} \beta \mapsto v,\left(1-(3 / 2) \rho_{2}\right) \beta \mapsto \widetilde{\beta}$,

$$
\begin{aligned}
u_{t}+u_{x} & +v u_{x x t}+\widetilde{\beta} u_{x x x}+\alpha u u_{x}+\frac{1}{3} v \alpha\left(u u_{x x x}+2 u_{x} u_{x x}\right) \\
& +3 \mu \alpha^{2} u^{2} u_{x}+v \mu \alpha^{2}\left(u^{2} u_{x x x}+u_{x}^{3}+4 u u_{x} u_{x x}\right) \\
& +v^{2} \mu \alpha^{2}\left(u_{x}^{2} u_{x x x}+2 u_{x} u_{x x}^{2}\right)=0
\end{aligned}
$$

From the above references and the references cited therein, we can know that (1) and (2) are very important water wave models. However, (1) and (2) are too complex to obtain their exact solution under universal conditions. Only under some special parametric conditions, their exact solutions were obtained in the existing literature. Next, let us briefly review the research backgrounds for the above equations.

In [2], Tzirtzilakis et al. only obtained two soliton-like solutions of (2) in the two groups of special conditions $\rho_{3}=$ $2\left(\rho_{1}-\rho_{2}\right), \rho_{4}=0, \rho_{5}=2 \rho_{1}\left(\rho_{2}-2 \rho_{1}\right), \rho_{6}=6 \rho_{1}\left(2 \rho_{1}-\rho_{2}\right)$, $\rho_{7}=3 \rho_{1}\left(\rho_{2}-2 \rho_{1}\right)$ and $\rho_{2}=2 \rho_{1}, \rho_{3}=-2 \rho_{1}, \rho_{3}=3 \rho_{4}$, $\rho_{6}=-6 \rho_{4}, \rho_{7}=3 \rho_{4}$. In [9], by using the planar bifurcation method of dynamical systems, under the four groups of special conditions $\left(A_{1}\right) \rho_{2}^{2}>4 \rho_{5}>0, \rho_{3}=2 \rho_{2}, \rho_{6}=4 \rho_{5}$, $\rho_{7}=\rho_{5} ;\left(A_{2}\right) \quad \rho_{3}=2 \rho_{2}, \rho_{5}=\rho_{7}=(1 / 4) \rho_{2}^{2}, \rho_{6}=(1 / 2) \rho_{1} \rho_{2}$; $\left(B_{1}\right) \rho_{3}=\rho_{2}, \rho_{6}=2 \rho_{5}, \rho_{7}=0 ;\left(B_{2}\right) \rho_{3}=\rho_{2}, \rho_{5}=(1 / 4) \rho_{2}^{2}$, $\rho_{6}=2 \rho_{5}=(1 / 2) \rho_{2}^{2}, \rho_{7}=0$, Li et al. studied (2); the existence of all kinds of traveling wave solutions were discussed completely, but its exact solutions were not investigated although some results of numerical simulation were obtained in this literature. In [10], in order to answer what is the dynamical behavior of one-loop soliton solution, Li studied the special case of (1) for $\rho_{1}=\rho_{4}=\rho_{6}=0, \rho_{3}=2 \rho_{2}$, $\rho_{5}=\rho_{7}=(1 / 4) \rho_{2}^{2}$. In [11], under different kinds of parametric conditions, Marinakis discussed two integrable cases for the third-order approximation model (1). In [12], Marinakis proved that (1) and some its of special cases are integrable. In [13], Gandarias and Bruzon proved that (1) is self-adjoint if and only if $\rho_{3}=2 \rho_{2}, \rho_{7}=\rho_{6}-3 \rho_{5}$. In [14], by using the method as in [9], Li et al. studied (10); the existence of solitary wave, kink, and antikink wave solutions and uncountably infinite many smooth and nonsmooth periodic wave solutions was discussed except exact solutions. In [8], Bi also obtained some results of numerical simulation of (10); it is a pity that the exact traveling wave solutions of (10) were not obtained yet. In $[4,5]$, we obtained some exact traveling wave solutions of (4) under the parametric conditions $\rho_{3}=(p+$ 1) $\rho_{2}$ or $\rho_{3}=p \rho_{2}$. In [15], under a new ansätze, Khuri studied (4); some exact solitary wave solutions and periodic wave solutions were obtained. In [16], by using method of planar dynamical system, Long et al. studied (6); the existence of smooth solitary wave and uncountably infinite many smooth and nonsmooth periodic wave solutions was proved in this literature.

From the above research backgrounds of (1) and (2), we can see that their exact solutions in universal conditions are 
hard to obtain because they are highly nonlinear equations and most probably they are not integrable equations in general. Thus, large numbers of research results are still concentrated in the classical $\mathrm{KdV}$ equation and some other high-order equations with $\mathrm{KdV}$ type, such as KdV-Burgers equation [17, 18] and KdV-Burgers-Kuramoto equation [19], at present. Therefore, the investigation of the more exact solutions for (1) is very important and necessary. However, by using the current methods, we can not obtain exact solutions of (1) in universal conditions; the next best thing is the investigation of exact solutions of (7). In this paper, still regarding the $\rho_{i}(i=1,2,3,4)$ as free parameters and by using the integral bifurcation method $[20,21]$, we will investigate exact traveling wave solutions and their properties of (7).

The rest of this paper is organized as follows. In Section 2, we will derive two-dimensional planar system which is equivalent to (7) and give its first integral equation. In Section 3, by using the integral bifurcation method, we will obtain some new traveling wave solutions and discuss their dynamic properties.

\section{Two-Dimensional Planar Dynamical System of (7) and Its First Integral and Conservation of Energy}

Making a transformation $\eta(t, x)=\phi(\xi)$ with $\xi=x-c t$, (7) can be reduced to the following ODE:

$$
\begin{aligned}
-c \phi^{\prime}+ & \phi^{\prime}+\alpha \phi \phi^{\prime}+\beta \phi^{\prime \prime \prime}+\rho_{1} \alpha^{2} \phi^{2} \phi^{\prime} \\
& +\alpha \beta\left(\rho_{2} \phi \phi^{\prime \prime \prime}+\rho_{3} \phi^{\prime} \phi^{\prime \prime}\right)+\rho_{4} \alpha^{3} \phi^{3} \phi^{\prime}=0,
\end{aligned}
$$

where $c$ is wave velocity which moves along the direction of $x$-axis and $c \neq 0$. Integrating (11) once and setting the integral constant as zero yields

$$
\begin{aligned}
& (1-c) \phi+\frac{1}{2} \alpha \phi^{2}+\beta \phi^{\prime \prime}+\frac{1}{3} \rho_{1} \alpha^{2} \phi^{3}+\alpha \beta \\
& \quad \times\left[\rho_{2} \phi \phi^{\prime \prime}+\frac{1}{2}\left(\rho_{3}-\rho_{2}\right)\left(\phi^{\prime}\right)^{2}\right]+\frac{1}{4} \rho_{4} \alpha^{3} \phi^{4}=0 .
\end{aligned}
$$

Let $\phi^{\prime}=y$. Thus (12) can be reduced to a planar system

$$
\begin{gathered}
\frac{d \phi}{d \xi}=y \\
\frac{d y}{d \xi}=-\left(\frac{1}{4} \rho_{4} \alpha^{3} \phi^{4}+\frac{1}{3} \rho_{1} \alpha^{2} \phi^{3}+\frac{1}{2} \alpha \phi^{2}+(1-c) \phi\right. \\
\left.+\frac{1}{2} \alpha \beta\left(\rho_{3}-\rho_{2}\right) y^{2}\right) \times\left(\beta\left(1+\alpha \rho_{2} \phi\right)\right)^{-1}
\end{gathered}
$$

and a linear equation

$$
\beta\left(1+\alpha \rho_{2} \phi\right)=0 .
$$

Obviously, the solutions of (12) cover the solutions of (13) and (14). We notice that the second equation in (13) is not continuous when $\phi=-1 / \alpha \rho_{2}$; that is, the function $\phi^{\prime \prime}(\xi)$ is not defined by $\phi=-1 / \alpha \rho_{2}$. So (13) is a singular system; the line $\phi=-1 / \alpha \rho_{2}$ is called the singular line. Thus, we make the following transformation:

$$
d \xi=12 \beta\left(1+\alpha \rho_{2} \phi\right) d \tau
$$

where $\tau$ is a free parameter. Under the transformation (15), (13) can be rewritten as the following system

$$
\begin{gathered}
\frac{d \phi}{d \tau}=12 \beta\left(1+\alpha \rho_{2} \phi\right) y \\
\frac{d y}{d \tau}=-\left[3 \rho_{4} \alpha^{3} \phi^{4}+4 \rho_{1} \alpha^{2} \phi^{3}+6 \alpha \phi^{2}+12(1-c) \phi\right. \\
\left.+6 \alpha \beta\left(\rho_{3}-\rho_{2}\right) y^{2}\right]
\end{gathered}
$$

Clearly, (16) is equivalent to (12). Except for the singular line $\phi=-1 / \alpha \rho_{2}$, (13) and (16) have the same first integral.

First, from the parametric conditions $\rho_{3}=5 / 3, \rho_{2}=$ $23 / 6$ in the model (1), we easily know that $\rho_{3} \approx 2 \rho_{2}$. Second, from [9-11, 13], we know that (1) contains many good properties including loop soliton, integrability, and self-adjoint property when $\rho_{3}=2 \rho_{2}$. In addition, in the condition $\rho_{3}=$ $2 \rho_{2}$, it becomes easy when we solve (7). Therefore, we only consider the special case of (7) for $\rho_{3}=2 \rho_{2}$ in this paper. Thus, by using this assumption, we obtain the first integral of (13) and (16) as follows:

$$
y^{2}=\frac{-(3 / 5) \alpha^{3} \rho_{4} \phi^{5}-\alpha^{2} \rho_{1} \phi^{4}-2 \alpha \phi^{3}+6(c-1) \phi^{2}+C_{0}}{6 \beta\left(1+\alpha \rho_{2} \phi\right)},
$$

where $C_{0}$ is an integral constant. For the convenience of discussion, taking the integral constant $C_{0}=0$ in (17) yields

$$
\begin{aligned}
\frac{1}{2} M y^{2} & +\frac{1}{2} M \alpha \rho_{2} \phi y^{2}+\frac{3}{5} \alpha^{3} \rho_{4} \phi^{5}+\alpha^{2} \rho_{1} \phi^{4} \\
& +2 \alpha \phi^{3}-6(c-1) \phi^{2}=0
\end{aligned}
$$

where $M=12 \beta$ denotes the particle's mass of system. Let $E_{1}=(1 / 2) M y^{2}=(1 / 2) M\left(\phi^{\prime}\right)^{2}, E_{2}=(1 / 2) M \alpha \rho_{2} \phi y^{2}=$ $(1 / 2) M \alpha \rho_{2} \phi\left(\phi^{\prime}\right)^{2}, T=(3 / 5) \alpha^{3} \rho_{4} \phi^{5}+\alpha^{2} \rho_{1} \phi^{4}+2 \alpha \phi^{3}-6(c-$ 1) $\phi^{2}$. Thus, (18) can be rewritten as the following equation of conservation of energy:

$$
E_{1}+E_{2}+T=0
$$

where $E_{1}$ denotes kinetic energy, $E_{2}$ denotes external energy, and $T$ denotes potential energy. In (19), the kinetic energy $E_{1}$ and potential energy $T$ are not conserved because the external energy $E_{2} \neq 0$, but the global energy $\left(E_{1}, E_{2}, T\right)$ are subject to the energy conservation. $E_{2}=0$ if only if $\rho_{2}=0$. Obviously, $\rho_{3}=0$ once $\rho_{2}=0$; under this case, (7) becomes the following equation:

$$
\eta_{t}+\eta_{x}+\alpha \eta \eta_{x}+\beta \eta_{x x x}+\rho_{1} \alpha^{2} \eta^{2} \eta_{x}+\rho_{4} \alpha^{3} \eta^{3} \eta_{x}=0 .
$$


Similarly, (20) can be reduced to the following planar system:

$$
\begin{gathered}
\frac{d \phi}{d \xi}=y \\
\frac{d y}{d \xi}=-\left(\frac{1}{4} \rho_{4} \alpha^{3} \phi^{4}+\frac{1}{3} \rho_{1} \alpha^{2} \phi^{3}+\frac{1}{2} \alpha \phi^{2}+(1-c) \phi\right) \times \beta^{-1}
\end{gathered}
$$

Obviously, the system (21) is a regular system; it has no singular line. Taking the integral constant as zero, we obtain (21)'s first integral as follows:

$$
y^{2}=-\frac{1}{\beta}\left[\frac{1}{10} \alpha^{3} \rho_{4} \phi^{5}+\frac{1}{6} \alpha^{2} \rho_{1} \phi^{4}+\frac{1}{3} \alpha \phi^{3}-(c-1) \phi^{2}\right] .
$$

Equation (22) can be rewritten as the following equation of conservation of energy:

$$
E_{1}+T=0
$$

where $E_{1}, T$ are given above. The kinetic energy $E_{1}$ and potential energy $T$ are conserved in (23); in other words, the particle motion satisfies the conservation of kinetic energy and potential energy, which converts the kinetic energy from the potential energy then it converts the potential energy from the kinetic energy and go round and round. Therefore, (20) only has nonsingular traveling wave solutions, and all solutions are smooth; this is very different from (7).

In order to discuss singular or nonsingular traveling wave solutions of (7), we will consider (17). When $C_{0}=0$, (17) can be reduced to

$$
\begin{aligned}
y= & \pm\left(\sqrt{\left[-\frac{3}{5} \alpha^{3} \rho_{4} \phi^{5}-\alpha^{2} \rho_{1} \phi^{4}-2 \alpha \phi^{3}+6(c-1) \phi^{2}\right]\left(1+\alpha \rho_{2} \phi\right)}\right) \\
& \times\left(\sqrt{6 \beta}\left(1+\alpha \rho_{2} \phi\right)\right)^{-1} .
\end{aligned}
$$

Equation (24) can be further simplified under the following parametric conditions.

Case 1. One has $c=1+1 / 3 \rho_{2}, \rho_{4}=-(5 / 3) \rho_{1} \rho_{2}$.

Case 2. One has $\rho_{1}=-2 \rho_{2}, \rho_{4}=(10 / 3) \rho_{2}^{2}$.

Case 3. One has $c=1+1 / 3 \rho_{2}, \rho_{1}=-2 \rho_{2}$.

Case 4. One has $c=1, \rho_{1}=-2 \rho_{2}$.

Case 5. One has $c \neq 1+1 / 3 \rho_{2}$ or $c \neq 1, \rho_{1}=-2 \rho_{2}, \rho_{4} \neq-$ $(5 / 3) \rho_{1} \rho_{2}$ or $\rho_{4} \neq(10 / 3) \rho_{2}^{2}$.

Under the parametric conditions of Case 1, (24) can be reduced to

$$
y= \pm \frac{\phi \sqrt{\alpha^{4} \rho_{1} \rho_{2}^{2} \phi^{4}-\alpha^{2}\left(\rho_{1}+2 \rho_{2}\right) \phi^{2}+2 / \rho_{2}}}{\sqrt{6 \beta}\left(1+\alpha \rho_{2} \phi\right)} .
$$

Under the parametric conditions of Case 2, (24) can be reduced to

$$
y= \pm \frac{\phi \sqrt{-2 \alpha^{4} \rho_{2}^{3} \phi^{4}+2 \alpha\left(3 c \rho_{2}-3 \rho_{2}-1\right) \phi+6(c-1)}}{\sqrt{6 \beta}\left(1+\alpha \rho_{2} \phi\right)} .
$$

Under the parametric conditions of Case 3, (24) can be reduced to

$$
y= \pm \frac{\phi \sqrt{-(3 / 5) \alpha^{4} \rho_{4} \rho_{2} \phi^{4}+(1 / 5) \alpha^{3}\left(10 \rho_{2}^{2}-3 \rho_{4}\right) \phi^{3}+2 / \rho_{2}}}{\sqrt{6 \beta}\left(1+\alpha \rho_{2} \phi\right)} .
$$

Under the parametric conditions of Case 4, (24) can be reduced to

$y=$

$$
\pm \frac{\phi \sqrt{-(3 / 5) \alpha^{4} \rho_{4} \rho_{2} \phi^{4}+(1 / 5) \alpha^{3}\left(10 \rho_{2}^{2}-3 \rho_{4}\right) \phi^{3}-2 \alpha \phi}}{\sqrt{6 \beta}\left(1+\alpha \rho_{2} \phi\right)} .
$$

Under the the parametric conditions of Case 5, (24) can be reduced to

$$
y= \pm \frac{\phi \sqrt{\left[-(3 / 5) \alpha^{3} \rho_{4} \phi^{3}-\alpha^{2} \rho_{1} \phi^{2}-2 \alpha \phi+6(c-1)\right]\left(1+\alpha \rho_{2} \phi\right)}}{\sqrt{6 \beta}\left(1+\alpha \rho_{2} \phi\right)} .
$$

\section{Exact Traveling Wave Solutions of (7) and Their Dynamic Properties}

In this section, by using (25), (26), (27), (28), and (29), we will derive many exact traveling wave solutions and discuss their dynamic properties.

3.1. The Exact Solutions under the Parametric Conditions of Case 1. Substituting (25) into the left expression (i.e., $\left.d \phi / d \tau=12 \beta\left(1+\alpha \rho_{2} \phi\right) y\right)$ of (16) yields

$$
\frac{d \phi}{\phi \sqrt{\alpha^{4} \rho_{1} \rho_{2}^{2} \phi^{4}-\alpha^{2}\left(\rho_{1}+2 \rho_{2}\right) \phi^{2}+2 / \rho_{2}}}= \pm 2 \sqrt{6 \beta} d \tau .
$$

For the sake of convenience, in all the following discussions, we only discuss the case where the right of equation is "+" sign; the case of "-" sign can be similarly discussed. By the way, the solutions obtained by taking "+" sign and the solutions obtained by taking "-" sign are same when the $\phi$ is even function.

Taking “+” in (30), it can be reduced to

$$
\frac{d \phi^{2}}{\phi^{2} \sqrt{\alpha^{4} \rho_{1} \rho_{2}^{2}\left(\phi^{2}\right)^{2}-\alpha^{2}\left(\rho_{1}+2 \rho_{2}\right) \phi^{2}+2 / \rho_{2}}}=4 \sqrt{6 \beta} d \tau \text {. }
$$


Let $\psi=\phi^{2}$. Equation (31) can be rewritten as

$$
\frac{d \psi}{\psi \sqrt{\alpha^{4} \rho_{1} \rho_{2}^{2} \psi^{2}-\alpha^{2}\left(\rho_{1}+2 \rho_{2}\right) \psi+2 / \rho_{2}}}=4 \sqrt{6 \beta} d \tau .
$$

Taking the $\left(0, \psi_{1,2}\right)$ as initial values, respectively, integrating (32) we obtain

$$
\begin{aligned}
& \int_{\psi_{1}}^{\psi} \frac{d \psi}{\psi \sqrt{\alpha^{4} \rho_{1} \rho_{2}^{2} \psi^{2}-\alpha^{2}\left(\rho_{1}+2 \rho_{2}\right) \psi+2 / \rho_{2}}} \\
& \quad=\int_{0}^{\tau} 4 \sqrt{6 \beta} d \tau, \\
& \int_{\psi_{2}}^{\psi} \frac{d \psi}{\psi \sqrt{\alpha^{4} \rho_{1} \rho_{2}^{2} \psi^{2}-\alpha^{2}\left(\rho_{1}+2 \rho_{2}\right) \psi+2 / \rho_{2}}} \\
& =\int_{0}^{\tau} 4 \sqrt{6 \beta} d \tau,
\end{aligned}
$$

where $\psi_{1,2}=\left(\rho_{1}+2 \rho_{2} \pm \sqrt{\left(\rho_{1}+2 \rho_{2}\right)^{2}-8 \rho_{1}}\right) / 2 \rho_{1} \rho_{2} \alpha^{2}$ are two roots of equation $\alpha^{4} \rho_{1} \rho_{2}^{2} \psi^{2}-\alpha^{2}\left(\rho_{1}+2 \rho_{2}\right) \psi+2 / \rho_{2}=0$.

(i) When $\rho_{2}>0, \Delta=\left(\rho_{1}+2 \rho_{2}\right)^{2}-8 \rho_{1}>0$, completing the integrals (33) yields

$$
\begin{aligned}
& \psi=\frac{4 R_{1}}{Q_{1}-P_{1} \cosh \left(8 \sqrt{\left(3 \beta / \rho_{2}\right)} \tau\right)}, \\
& \psi=\frac{4 R_{2}}{P_{2} \cosh \left(8 \sqrt{\left(3 \beta / \rho_{2}\right)}\right)-Q_{2}},
\end{aligned}
$$

where $R_{1,2}=\left(\rho_{1}+2 \rho_{2} \pm \sqrt{\Delta}\right)\left[\left(\rho_{1}+2 \rho_{2}\right) \sqrt{\Delta} \pm \Delta\right] / 2 \rho_{1}^{2} \rho_{2}^{3} \alpha^{2}$, $Q_{1,2}=\left(\rho_{1}+2 \rho_{2}\right)\left[\sqrt{\Delta} \pm\left(\rho_{1}+2 \rho_{2}\right)\right]\left[\left(\rho_{1}+2 \rho_{2}\right) \sqrt{\Delta} \pm\left(\rho_{1}^{2}+\right.\right.$ $\left.\left.4 \rho_{2}^{2}\right)\right] / 2 \rho_{1}^{2} \rho_{2}^{2}$, and $P_{1,2}=\Delta\left[\sqrt{\Delta} \pm\left(\rho_{1}+2 \rho_{2}\right)\right]^{2} / 4 \rho_{1}^{2} \rho_{2}^{2}$ with $\Delta=$ $\left(\rho_{1}+2 \rho_{2}\right)^{2}-8 \rho_{1}$.

By using (34) and the transformation $\phi^{2}=\psi$, we obtain four exact solutions of (31) as follows:

$$
\begin{aligned}
& \phi= \pm \frac{2 \sqrt{R_{1}}}{\sqrt{Q_{1}-P_{1} \cosh \left(8 \sqrt{\left(3 \beta / \rho_{2}\right) \tau}\right)}}, \\
& \phi= \pm \frac{2 \sqrt{R_{2}}}{\sqrt{P_{2} \cosh \left(8 \sqrt{\left(3 \beta / \rho_{2}\right) \tau}\right)-Q_{2}}},
\end{aligned}
$$

where the constants $R_{1,2}, P_{1,2}, Q_{1,2}$ are given above.

Substituting (35) into (15) yields

$$
\begin{aligned}
\xi= & 12 \beta \pm \alpha \rho_{2} g_{1} \sqrt{3 \rho_{2} \beta R_{1}} \\
& \times F\left(\arcsin \sqrt{\frac{Q_{1}-P_{1} \cosh \left(8 \sqrt{\left(3 \beta / \rho_{2}\right)}\right)}{Q_{1}-P_{1}}}, \sqrt{\frac{\mathrm{Q}_{1}-\mathrm{P}_{1}}{\mathrm{Q}_{1}+\mathrm{P}_{1}}}\right),
\end{aligned}
$$

where $F(\varphi, k)$ is a normal elliptic integral of the first kind and $g_{1}=2 / \sqrt{Q_{1}+P_{1}}, Q_{1}>P_{1}>0,0<\tau<(1 / 8) \sqrt{\left(\rho_{2} / 3 \beta\right)}$ $\cosh ^{-1}\left(Q_{1} / P_{1}\right)$.

Substituting (36) into (15) yields

$$
\begin{aligned}
\xi= & 12 \beta \pm \alpha \rho_{2} g_{2} \sqrt{3 \rho_{2} \beta R_{2}} \\
& \times F\left(\arcsin \sqrt{\frac{P_{2}\left(\cosh \left(8 \sqrt{\left(3 \beta / \rho_{2}\right)} \tau\right)-1\right)}{P_{2} \cosh \left(8 \sqrt{\left(3 \beta / \rho_{2}\right)} \tau\right)-Q_{2}}}, \sqrt{\frac{P_{2}+Q_{2}}{2 P_{2}}}\right),
\end{aligned}
$$

where $g_{2}=\sqrt{2 / \rho_{2}}, P_{2}>Q_{2}>0$, and $0<\tau<+\infty$.

Combining (35) with (37), we obtain (7)'s two exact solutions of parametric type as follows:

$$
\begin{gathered}
\eta=\phi= \pm \frac{2 \sqrt{R_{1}}}{\sqrt{Q_{1}-P_{1} \cosh \left(8 \sqrt{\left(3 \beta / \rho_{2}\right)} \tau\right)}}, \\
\xi=12 \beta \tau \pm \alpha \rho_{2} g_{1} \sqrt{3 \rho_{2} \beta R_{1}} \\
\times F\left(\arcsin \sqrt{\frac{Q_{1}-P_{1} \cosh \left(8 \sqrt{\left(3 \beta / \rho_{2}\right)}\right)}{Q_{1}-P_{1}}}, \sqrt{\frac{Q_{1}-P_{1}}{Q_{1}+P_{1}}}\right) .
\end{gathered}
$$

Combining (36) with (38), we obtain (7)'s another two exact solutions of parametric type as follows:

$$
\begin{gathered}
\eta=\phi= \pm \frac{2 \sqrt{R_{2}}}{\sqrt{P_{2} \cosh \left(8 \sqrt{\left(3 \beta / \rho_{2}\right)}\right)-Q_{2}}}, \\
\xi=12 \beta \tau \pm \alpha \rho_{2} g_{2} \sqrt{3 \rho_{2} \beta R_{2}} \\
\times F\left(\arcsin \sqrt{\frac{P_{2}\left(\cosh \left(8 \sqrt{\left(3 \beta / \rho_{2}\right)}\right)-1\right)}{P_{2} \cosh \left(8 \sqrt{\left(3 \beta / \rho_{2}\right)}\right)-Q_{2}}}, \sqrt{\frac{P_{2}+Q_{2}}{2 P_{2}}}\right) .
\end{gathered}
$$

(ii) When $\rho_{2}<0, \Delta=\left(\rho_{1}+2 \rho_{2}\right)^{2}-8 \rho_{1}>0$, completing the integrals (33) yields

$$
\begin{aligned}
& \psi=\frac{4}{-\alpha^{2} \rho_{2} \sqrt{\Delta}\left[-\alpha^{2}\left(\rho_{1}+2 \rho_{2}\right)-\cos \left(8 \sqrt{-\left(3 \beta / \rho_{2}\right)} \tau\right)\right]}, \\
& \psi=\frac{4}{-\alpha^{2} \rho_{2} \sqrt{\Delta}\left[-\alpha^{2}\left(\rho_{1}+2 \rho_{2}\right)+\cos \left(8 \sqrt{-\left(3 \beta / \rho_{2}\right)} \tau\right)\right]}
\end{aligned}
$$


Similarly, by using (41) and the transformation $\phi^{2}=\psi$, we obtain four periodic solutions of (31) as follows:

$$
\phi= \pm \frac{2}{\sqrt{-\alpha^{2} \rho_{2} \sqrt{\Delta}} \sqrt{-\alpha^{2}\left(\rho_{1}+2 \rho_{2}\right)-\cos \left(8 \sqrt{-\left(3 \beta / \rho_{2}\right)} \tau\right)}}
$$

$$
\phi= \pm \frac{2}{\sqrt{-\alpha^{2} \rho_{2} \sqrt{\Delta}} \sqrt{-\alpha^{2}\left(\rho_{1}+2 \rho_{2}\right)+\cos \left(8 \sqrt{-\left(3 \beta / \rho_{2}\right)} \tau\right)}}
$$

where $\rho_{1}<-\left(2 \rho_{2}+1 / \alpha^{2}\right)$; that is, $-\alpha^{2}\left(\rho_{1}+2 \rho_{2}\right)>1>0$. Respectively, substituting (42) and (43) into (15) yields

$$
\begin{gathered}
\xi=12 \beta \tau \pm \frac{\tilde{g} \rho_{2} \sqrt{3 \beta}}{\sqrt[4]{\Delta}} \\
\times F\left(\arcsin \sqrt{\frac{\left[1-\cos \left(8 \sqrt{-\left(3 \beta / \rho_{2}\right)} \tau\right)\right]\left[\alpha^{2}\left(\rho_{1}+2 \rho_{2}\right)-1\right]}{2\left[\alpha^{2}\left(\rho_{1}+2 \rho_{2}\right)+\cos \left(8 \sqrt{-\left(3 \beta / \rho_{2}\right)} \tau\right)\right]}}, k\right) \\
\xi=12 \beta \tau \pm \frac{\tilde{g} \rho_{2} \sqrt{3 \beta}}{\sqrt[4]{\Delta}} F\left(4 \sqrt{\left.-\frac{3 \beta}{\rho_{2}} \tau, k\right)}\right.
\end{gathered}
$$

where $\tilde{g}=2 / \sqrt{1-\alpha^{2}\left(\rho_{1}+2 \rho_{2}\right)}, k=\sqrt{2 /\left(1-\alpha^{2}\left(\rho_{1}+2 \rho_{2}\right)\right)}$.

Combining (42) with (44), we obtain (7)'s two exact solutions of parametric type as follows:

$$
\begin{gathered}
\eta=\phi= \pm \frac{2}{\sqrt{-\alpha^{2} \rho_{2} \sqrt{\Delta}} \sqrt{-\alpha^{2}\left(\rho_{1}+2 \rho_{2}\right)-\cos \left(8 \sqrt{-\left(3 \beta / \rho_{2}\right)} \tau\right)}} \\
\xi=12 \beta \tau \pm \frac{\tilde{g} \rho_{2} \sqrt{3 \beta}}{\sqrt[4]{\Delta}} \\
\quad \times F\left(\arcsin \sqrt{\frac{\left[1-\cos \left(8 \sqrt{-\left(3 \beta / \rho_{2}\right)} \tau\right)\right]\left[\alpha^{2}\left(\rho_{1}+2 \rho_{2}\right)-1\right]}{2\left[\alpha^{2}\left(\rho_{1}+2 \rho_{2}\right)+\cos \left(8 \sqrt{-\left(3 \beta / \rho_{2}\right)} \tau\right)\right]}}, k\right),
\end{gathered}
$$

where $0<\tau \leq \pi \sqrt{-\rho_{2}} / 8 \sqrt{3 \beta}$.

Combining (43) with (45), we obtain (7)'s another two exact solutions of parametric type as follows:

$$
\begin{aligned}
& \eta=\phi \\
& = \pm \frac{2}{\sqrt{-\alpha^{2} \rho_{2} \sqrt{\Delta}} \sqrt{-\alpha^{2}\left(\rho_{1}+2 \rho_{2}\right)+\cos \left(8 \sqrt{-\left(3 \beta / \rho_{2}\right)} \tau\right)}}, \\
& \quad \xi=12 \beta \tau \pm \frac{\tilde{g} \rho_{2} \sqrt{3 \beta}}{\sqrt[4]{\Delta}} F\left(4 \sqrt{\left.-\frac{3 \beta}{\rho_{2}} \tau, k\right) .}\right.
\end{aligned}
$$

Among the above traveling wave solutions, it is very worthy to mention solutions (40), (46), and (47); they have some peculiar dynamical properties and their traveling wave phenomena are very interesting. Solution (40) denotes a singular solitary wave; its shape is very similar to bright soliton, but it is not a normal soliton; the left parts of waveform degenerate into a crook. Solution (46) denotes a bounded wave with level asymptote; its shape is a half of a whole loop soliton; we call it semiloop soliton. In order to describe the dynamic properties of these two traveling wave solutions intuitively, we, respectively, plot profile of solutions (40) and (46) by using software Maple, which are shown in Figure 1.

Figure 1(a) shows a shape of singular solitary wave under the fixed parameters $\alpha=0.5, \beta=0.4, \rho_{1}=300, \rho_{2}=1.3$. Figure 1(b) shows a shape of semiloop soliton under the fixed parameters $\alpha=0.5, \beta=0.4, \rho_{1}=0.5, \rho_{2}=-4$.

It is very interesting that the solution (47) has six kinds of waveforms. Solution (47) with “+”, respectively, denotes a dark peakon, a dark loop soliton, and a broken loop soliton when the parameter $\rho_{1}$ decreases from 1.55 to -8.25 , which are shown in Figures 2(a)-2(d). Solution (47) with “-", respectively, denotes a bright soliton and a singular compacton when the parameter $\rho_{1}$ decreases from 1.65 to -6.25 , which are shown in Figures 2(e) and 2(f). In other words, its waveforms depend on parameter $\rho_{1}$ extremity. Similar traveling wave phenomena that one solution contains multiwaveform first appeared in the investigation of the Degasperis-Procesi equation [22].

Figure 2(a) shows a shape of dark soliton under the fixed parameters $\alpha=0.4, \beta=0.1, \rho_{2}=-4$, and $\rho_{1}=1.55$. Figure 2(b) shows a shape of dark peakon under the fixed parameters $\alpha=0.4, \beta=0.1, \rho_{2}=-4$, and $\rho_{1}=0.479$. Figure 2(c) shows a shape of dark loop soliton under the fixed parameters $\alpha=0.4, \beta=0.1, \rho_{2}=-4$, and $\rho_{1}=$ 0.05. Similar traveling wave phenomena also appear in [5], in "Concluding remarks" of this literature; by using these phenomena, we successfully explain the movement of water waves. Figure 2(d) shows a shape of broken wave of C-form (upward hatch $\mathrm{C}$ ) under the fixed parameters $\alpha=0.4, \beta=$ $0.1, \rho_{2}=-4$, and $\rho_{1}=-8.25$; we also call it broken loop soliton or open-orbicular wave. Figure 2(e) shows a shape of bright soliton under the fixed parameters $\alpha=0.4, \beta=0.1$, $\rho_{2}=-4$, and $\rho_{1}=1.65$. Figure 2(f) shows a shape of singular compacton under the fixed parameters $\alpha=0.4, \beta=0.1$, $\rho_{2}=-4$, and $\rho_{1}=-6.25$; we call it singular compacton because it is not a normal compacton. Specifically, both sides of compacton wave become upward crook; it very much likes solitary waves but it is not a solitary wave after all because the left and right parts of waveform do not extend as $\xi \rightarrow \infty$.

3.2. The Exact Solutions under the Parametric Conditions of Case 2. Notice that the characters of (26)-(29) are quite different from (25). Therefore, by using the expression $d \phi / d \xi=$ $y$ and (26)-(29), we will investigate the implicit solutions of (7) next. 


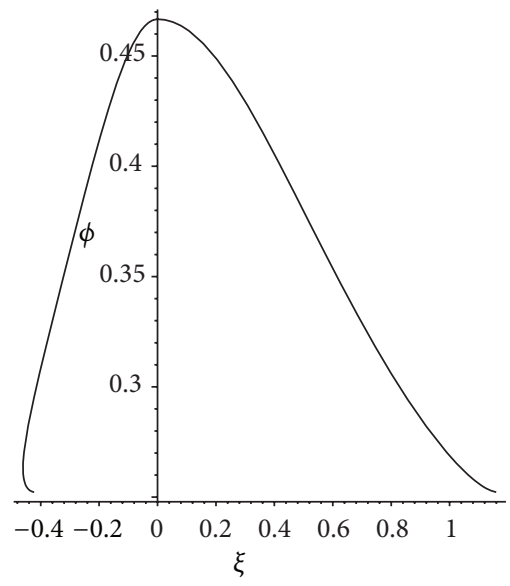

(a) Singular solitary wave

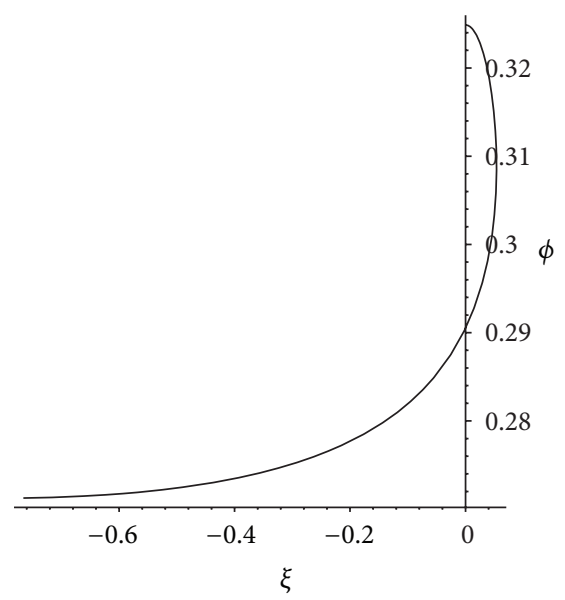

(b) Semiloop soliton

FIGURE 1: Profiles of solutions (40) and (46) for the fixed parameters. yields

Substituting (26) into the expression $d \phi / d \xi=y$ of (13)

$$
\frac{d \phi}{d \xi}= \pm \frac{\phi \sqrt{-2 \alpha^{4} \rho_{2}^{3} \phi^{4}+2 \alpha\left(3 c \rho_{2}-3 \rho_{2}-1\right) \phi+6(c-1)}}{\sqrt{6 \beta}\left(1+\alpha \rho_{2} \phi\right)}
$$

Taking “+”, (48) can be reduced to

$$
\begin{gathered}
\frac{\left(1+\alpha \rho_{2} \phi\right) d \phi}{\phi \sqrt{-2 \alpha^{4} \rho_{2}^{3} \phi^{4}+2 \alpha\left(3 c \rho_{2}-3 \rho_{2}-1\right) \phi+6(c-1)}} \\
=\frac{1}{\sqrt{6 \beta}} d \xi .
\end{gathered}
$$

(i) When $\rho_{2}<0$, (49) can be reduced to

$$
\begin{gathered}
\left(1+\alpha \rho_{2} \phi\right) d \phi\left(\phi \sqrt{\phi^{4}-\frac{3 c \rho_{2}-3 \rho_{2}-1}{\alpha^{3} \rho_{2}^{3}} \phi-\frac{3(c-1)}{\alpha^{4} \rho_{2}^{3}}}\right)^{-1} \\
=-\alpha^{2} \rho_{2} \sqrt{-\frac{\rho_{2}}{3 \beta}} d \xi
\end{gathered}
$$

By the Ferrari method or the Descartes method, it is easy to know that the equation

$$
\phi^{4}-\frac{3 c \rho_{2}-3 \rho_{2}-1}{\alpha^{3} \rho_{2}^{3}} \phi-\frac{3(c-1)}{\alpha^{4} \rho_{2}^{3}}=0
$$

has four roots as follows:

$$
\begin{aligned}
& -\frac{1}{\alpha \rho_{2}}, \quad \frac{(1 / 6) \sqrt[3]{\Delta_{1}}-4 / 3 \sqrt[3]{\Delta_{1}}+1 / 3}{\alpha \rho_{2}} \\
& \frac{-(1 / 12) \sqrt[3]{\Delta_{1}}+2 / 3 \sqrt[3]{\Delta_{1}}+1 / 3}{\alpha \rho_{2}} \\
& \pm \frac{i(\sqrt{3} / 2)\left((1 / 6) \sqrt[3]{\Delta_{1}}+4 / 3 \sqrt[3]{\Delta_{1}}\right)}{\alpha \rho_{2}}
\end{aligned}
$$

where $\Delta_{1}=-28-324 \rho_{2}(c-1)+36 \sqrt{\Delta_{2}}$ with $\Delta_{2}=81(1-$ c) $)^{2} \rho_{2}^{2}+14(1-c) \rho_{2}+1$. Especially, when $c=1$, these four roots can be reduced to $-1 / \alpha \rho_{2}, 0,(1 \pm \sqrt{3} i) / 2 \alpha \rho_{2}$. For the convenience of discussion, we denote the above two real roots by the signs $\nu, \gamma$ and always assume that the biggest root is denoted by $v$. We also denote the above two complex roots by the signs $s, \bar{s}$. Thus (50) can be rewritten as

$$
\begin{gathered}
\frac{d \phi}{\phi \sqrt{(\phi-\nu)(\phi-\gamma)(\phi-s)(\phi-\bar{s})}} \\
+\frac{\alpha \rho_{2} d \phi}{\sqrt{(\phi-\nu)(\phi-\gamma)(\phi-s)(\phi-\bar{s})}} \\
=-\alpha^{2} \rho_{2} \sqrt{-\frac{\rho_{2}}{3 \beta}} d \xi,
\end{gathered}
$$

where $v>\gamma$. Taking $(\nu, 0)$ as the initial values and integrating (53), we obtain implicit solution of (7) as follows:

$$
\begin{aligned}
& \frac{(\widetilde{B}-\widetilde{A})}{\widetilde{B} v+\gamma \widetilde{A}}\left[\mu_{1} F(\varphi, k)+\frac{\mu-\mu_{1}}{1-\mu^{2}}\left(\Pi\left(\varphi, \frac{\mu^{2}}{\mu^{2}-1}, k\right)-\mu f_{1}\right)\right] \\
& \quad+g \alpha \rho_{2} F(\varphi, k)=-\alpha^{2} \rho_{2} \sqrt{-\frac{\rho_{2}}{3 \beta}} \xi
\end{aligned}
$$




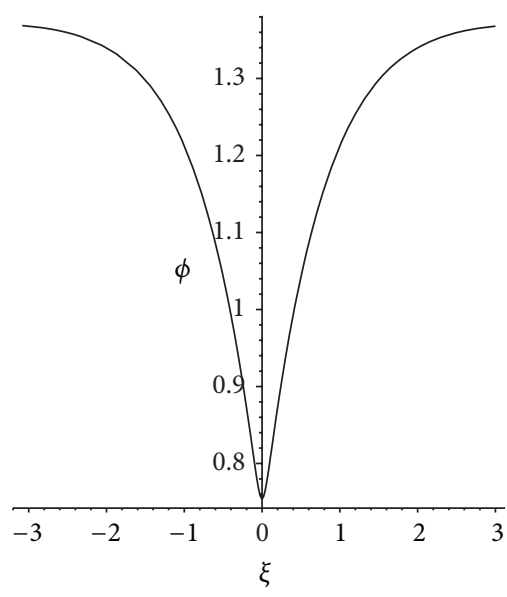

(a) Dark soliton

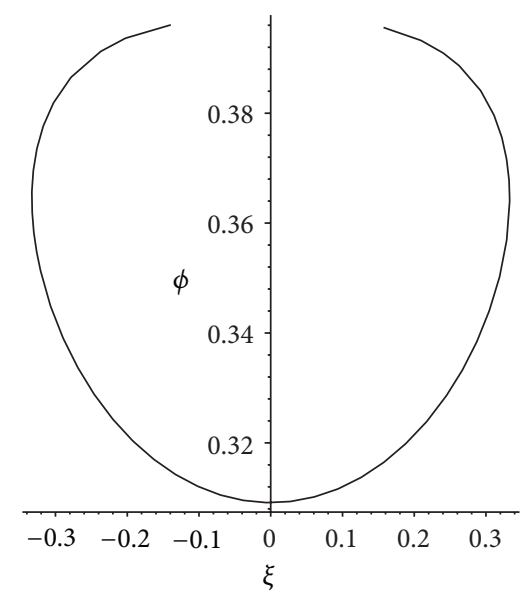

(d) Broken loop soliton (broken wave of Cform)

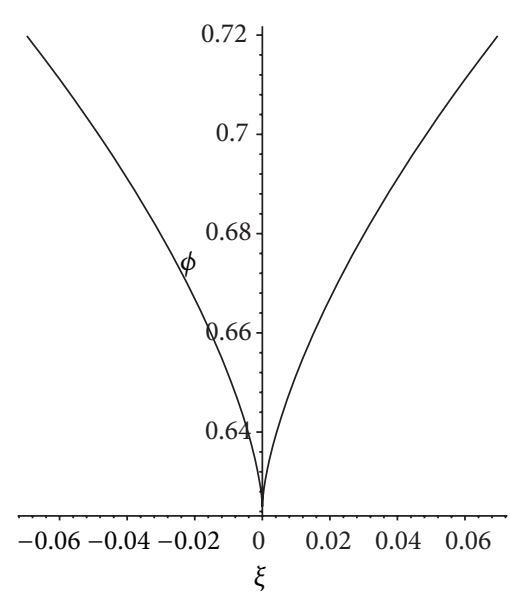

(b) Dark peakon

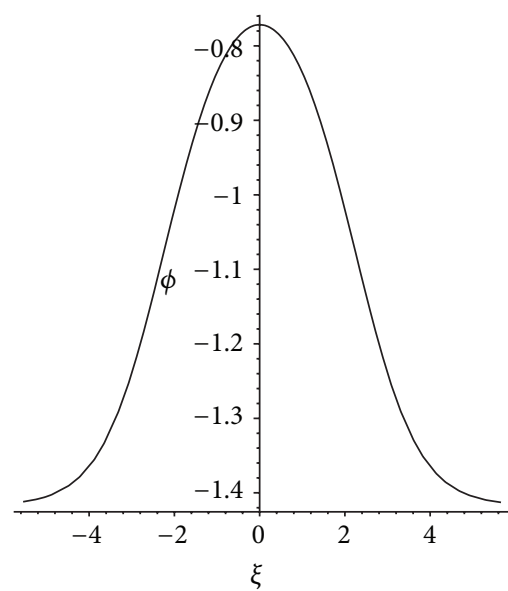

(e) Bright soliton

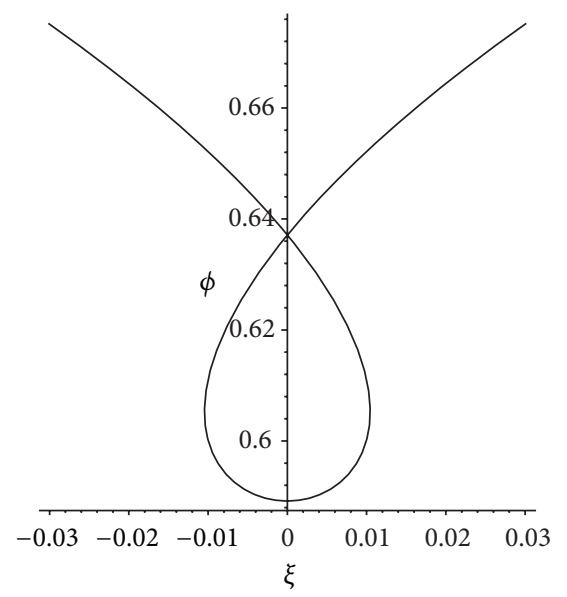

(c) Dark loop soliton

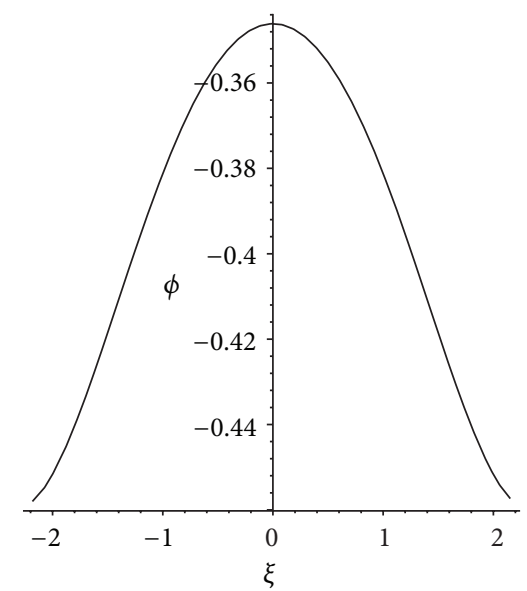

(f) Singular compacton

FIGURE 2: Different kinds of waveforms on solution (47) under different parametric conditions.

where $\widetilde{A}^{2}=(v-(s+\bar{s}) / 2)^{2}-(s-\bar{s})^{2} / 4, \widetilde{B}^{2}=(\gamma-(s+\bar{s}) / 2)^{2}-$

$$
\begin{aligned}
& (s-\bar{s})^{2} / 4, g=1 / \sqrt{\widetilde{A} \widetilde{B}}, k^{2}=\left((\widetilde{A}+\widetilde{B})^{2}-(v-\gamma)^{2}\right) / 4 \widetilde{A} \widetilde{B}, \\
& \varphi=\arccos \left[\frac{(\widetilde{A}-\widetilde{B}) \phi+\nu \widetilde{B}-\gamma \widetilde{A}}{(\widetilde{A}+\widetilde{B}) \phi-v \widetilde{B}-\gamma \widetilde{A}}\right], \\
& \mu=\frac{\gamma \widetilde{A}+\nu \widetilde{B}}{\nu \widetilde{B}-\gamma \widetilde{A}}, \quad \mu_{1}=\frac{\widetilde{A}+\widetilde{B}}{\widetilde{B}-\widetilde{A}} \\
& f_{1}=\sqrt{\frac{1-\mu^{2}}{k^{2}+k^{\prime 2} \mu^{2}}} \arctan \left[\varphi \sqrt{\frac{k^{2}+k^{\prime 2} \mu^{2}}{\left(1-\mu^{2}\right)\left(1-k^{2} \varphi^{2}\right)}}\right], \\
& \text { if } \frac{\mu^{2}}{\mu^{2}-1}<k^{2} \\
& =\frac{\varphi}{\sqrt{1-k^{2} \varphi^{2}}}, \quad \text { if } \frac{\mu^{2}}{\mu^{2}-1}=k^{2}
\end{aligned}
$$$$
\begin{gathered}
=\frac{1}{2} \sqrt{\frac{\mu^{2}-1}{k^{2}+k^{\prime 2} \mu^{2}}} \\
\times \ln \left[\frac{\sqrt{\left(k^{2}+k^{\prime 2} \mu^{2}\right)\left(1-k^{2} \varphi^{2}\right)}+\varphi \sqrt{\mu^{2}-1}}{\left(\sqrt{\left.k^{2}+k^{\prime 2} \mu^{2}\right)\left(1-k^{2} \varphi^{2}\right)}-\varphi \sqrt{\mu^{2}-1}\right.}\right], \\
\text { if } \frac{\mu^{2}}{\mu^{2}-1}>k^{2} .
\end{gathered}
$$

(ii) When $\rho_{2}>0$, (49) can be reduced to

$$
\begin{aligned}
& \frac{\left(1+\alpha \rho_{2} \phi\right) d \phi}{\phi \sqrt{-\phi^{4}+\left(\left(3 c \rho_{2}-3 \rho_{2}-1\right) /\left(\alpha^{3} \rho_{2}^{3}\right)\right) \phi+3(c-1) / \alpha^{4} \rho_{2}^{3}}} \\
& =\alpha^{2} \rho_{2} \sqrt{\frac{\rho_{2}}{3 \beta}} d \xi .
\end{aligned}
$$


Similarly, (56) can be rewritten as

$$
\begin{aligned}
& \frac{d \phi}{\phi \sqrt{(\nu-\phi)(\phi-\gamma)(\phi-s)(\phi-\bar{s})}} \\
& +\frac{\alpha \rho_{2} d \phi}{\sqrt{(\nu-\phi)(\phi-\gamma)(\phi-s)(\phi-\bar{s})}} \\
& =\alpha^{2} \rho_{2} \sqrt{\frac{\rho_{2}}{3 \beta}} d \xi,
\end{aligned}
$$

where $\nu, \gamma s, \widetilde{s}$ are four roots of (51) and $\nu \geq \phi>\gamma$. Taking $(\gamma, 0)$ as the initial values then integrating $(56)$, we obtain another implicit solution of (7) as follows:

$$
\begin{aligned}
& \frac{(\widetilde{A}+\widetilde{B})}{\gamma \widetilde{A}-\widetilde{B} v}\left[\mu_{2} F(\widetilde{\varphi}, m)+\frac{\tilde{\mu}-\mu_{2}}{1-\widetilde{\mu}^{2}}\right. \\
& \left.\quad \times\left(\Pi\left(\widetilde{\varphi}, \frac{\tilde{\mu}^{2}}{\widetilde{\mu}^{2}-1}, m\right)-\widetilde{\mu} f_{2}\right)\right]+\alpha \rho_{2} g F(\widetilde{\varphi}, m) \\
& =\alpha^{2} \rho_{2} \sqrt{\frac{\rho_{2}}{3 \beta}} \xi,
\end{aligned}
$$

where the constants $\widetilde{A}, \widetilde{B}, \widetilde{g}$ are given above, the $\widetilde{\varphi}=$ $\arccos [(\widetilde{B}(\nu-\phi)-\widetilde{A}(\phi-\gamma)) /(\widetilde{B}(\nu-\phi)+\widetilde{A}(\phi-\gamma))], m^{2}=$ $\left((\nu-\gamma)^{2}-(\widetilde{A}-\widetilde{B})^{2}\right) / 4 \widetilde{A} \widetilde{B}, \widetilde{\mu}=(\gamma \widetilde{A}-v \widetilde{B}) /(\gamma \widetilde{A}+v \widetilde{B}), \mu_{2}=$ $(\widetilde{A}-\widetilde{B}) /(\widetilde{A}+\widetilde{B})$, and

$$
\begin{aligned}
& f_{2}=\sqrt{\frac{1-\tilde{\mu}^{2}}{m^{2}+m^{\prime 2} \tilde{\mu}^{2}}} \arctan \left[\tilde{\varphi} \sqrt{\frac{m^{2}+m^{\prime 2} \widetilde{\mu}^{2}}{\left(1-\widetilde{\mu}^{2}\right)\left(1-m^{2} \widetilde{\varphi}^{2}\right)}}\right], \\
& \text { if } \frac{\tilde{\mu}^{2}}{\widetilde{\mu}^{2}-1}<m^{2} \\
& =\frac{\widetilde{\varphi}}{\sqrt{1-m^{2} \widetilde{\varphi}^{2}}}, \quad \text { if } \frac{\tilde{\mu}^{2}}{\widetilde{\mu}^{2}-1}=m^{2} \\
& =\frac{1}{2} \sqrt{\frac{\tilde{\mu}^{2}-1}{m^{2}+m^{\prime 2} \tilde{\mu}^{2}}} \\
& \times \ln \left[\frac{\sqrt{\left(m^{2}+m^{\prime 2} \tilde{\mu}^{2}\right)\left(1-m^{2} \widetilde{\varphi}^{2}\right)}+\tilde{\varphi} \sqrt{\tilde{\mu}^{2}-1}}{\sqrt{\left(m^{2}+m^{\prime 2} \tilde{\mu}^{2}\right)\left(1-m^{2} \tilde{\varphi}^{2}\right)}-\tilde{\varphi} \sqrt{\tilde{\mu}^{2}-1}}\right], \\
& \text { if } \frac{\tilde{\mu}^{2}}{\widetilde{\mu}^{2}-1}>m^{2} \text {. }
\end{aligned}
$$

3.3. The Exact Solutions under the Parametric Conditions of Case 3. As in Section 3.2, substituting (27) into the expression $d \phi / d \xi=y$ of (13) yields

$$
\begin{aligned}
\frac{d \phi}{d \xi}= & \pm\left(\phi \sqrt{-\frac{3}{5} \alpha^{4} \rho_{4} \rho_{2} \phi^{4}+\frac{1}{5} \alpha^{3}\left(10 \rho_{2}^{2}-3 \rho_{4}\right) \phi^{3}+\frac{2}{\rho_{2}}}\right) \\
& \times\left(\sqrt{6 \beta}\left(1+\alpha \rho_{2} \phi\right)\right)^{-1} .
\end{aligned}
$$

Taking “+”, (60) can be rewritten as

$$
\begin{aligned}
& \frac{\left(1+\alpha \rho_{2} \phi\right) d \phi}{\phi \sqrt{-(3 / 5) \alpha^{4} \rho_{4} \rho_{2} \phi^{4}+(1 / 5) \alpha^{3}\left(10 \rho_{2}^{2}-3 \rho_{4}\right) \phi^{3}+2 / \rho_{2}}} \\
& =\frac{1}{\sqrt{6 \beta}} d \xi .
\end{aligned}
$$

When $\rho_{4} \rho_{2}<0$, (61) can be reduced to

$$
\begin{gathered}
\frac{\left(1+\alpha \rho_{2} \phi\right) d \phi}{\phi \sqrt{\phi^{4}-\left(\left(10 \rho_{2}^{2}-3 \rho_{4}\right) / 3 \alpha \rho_{4} \rho_{2}\right) \phi^{3}-\left(10 / 3 \alpha^{4} \rho_{4} \rho_{2}^{2}\right)}} \\
=\alpha^{2} \sqrt{-\frac{\rho_{4} \rho_{2}}{10 \beta}} d \xi .
\end{gathered}
$$

When $\rho_{4} \neq 10 \rho_{2}^{2} / 3$, the equation

$$
\phi^{4}-\frac{10 \rho_{2}^{2}-3 \rho_{4}}{3 \alpha \rho_{4} \rho_{2}} \phi^{3}-\frac{10}{3 \alpha^{4} \rho_{4} \rho_{2}^{2}}=0
$$

has four roots as follows:

$$
\begin{gathered}
-\frac{1}{\alpha \rho_{2}}, \quad \frac{\left(1 / 9 \rho_{2}\right) \sqrt[3]{\Delta_{3}}+10 \rho_{2}\left(10 \rho_{2}^{2}-9 \rho_{4}\right) / 9 \sqrt[3]{\Delta_{3}}+10 \rho_{2} / 9}{\alpha \rho_{4}}, \\
\left(\left(-\frac{1}{18 \rho_{2}} \sqrt[3]{\Delta_{3}}-\frac{5 \rho_{2}\left(10 \rho_{2}^{2}-9 \rho_{2}\right)}{9 \sqrt[3]{\Delta_{3}}}+\frac{10 \rho_{2}}{9}\right)\right. \\
\left. \pm i \frac{\sqrt{3}}{2}\left(\frac{1}{9 \rho_{2}} \sqrt[3]{\Delta_{3}}-\frac{10 \rho_{2}\left(10 \rho_{2}^{2}-9 \rho_{4}\right)}{9 \sqrt[3]{\Delta_{3}}}\right)\right) \times\left(\alpha \rho_{4}\right)^{-1}
\end{gathered}
$$

where $\Delta_{3}=\rho_{2}^{2}\left(-1350 \rho_{4} \rho_{2}^{2}+1215 \rho_{4}^{2}+1000 \rho_{2}^{2}+135 \rho_{4} \sqrt{\Delta_{4}}\right)$ with $\Delta_{4}=-140 \rho_{4} \rho_{2}^{2}+100 \rho_{2}^{4}+81 \rho_{4}^{2}$. When $\rho_{4}=10 \rho_{2}^{2} / 3$, (63) has another four roots $\pm 1 / \alpha \rho_{2}, \pm\left(1 / \alpha \rho_{2}\right) i$.

(i) Particularly, when $\rho_{2}<0$ and $\rho_{4}=10 \rho_{2}^{2} / 3$, (62) can be reduced to

$$
\frac{\left(1+\alpha \rho_{2} \phi\right)}{\phi \sqrt{\phi^{4}-\left(1 / \alpha^{4} \rho_{2}^{4}\right)}} d \phi=-\alpha^{2} \rho_{2} \sqrt{-\frac{\rho_{2}}{3 \beta}} d \xi
$$

According to the above cases, we find that (63) still has two real roots and two complex roots; this case is very similar to that in (51) except their especial cases are different. Therefore, the types of their solutions are the same when $\rho_{4} \rho_{2}<0$ 
and $\rho_{4} \neq 10 \rho_{2}^{2} / 3$. Thus, we omit these parts of discussions. The especial case of (62) (i.e., (65)) can be reduced to

$$
\begin{gathered}
\frac{1}{2} \frac{d \phi^{2}}{\phi^{2} \sqrt{\left(\phi^{2}\right)^{2}-\left(1 / \alpha^{2} \rho_{2}^{2}\right)^{2}}}+\frac{\alpha \rho_{2} d \phi}{\sqrt{\left(\phi^{2}+1 / \alpha^{2} \rho_{2}^{2}\right)\left(\phi^{2}-1 / \alpha^{2} \rho_{2}^{2}\right)}} \\
=-\alpha^{2} \rho_{2} \sqrt{-\frac{\rho_{2}}{3 \beta}} d \xi
\end{gathered}
$$

where $\phi>1 / \alpha^{2} \rho_{2}^{2}>0$. Taking the $\left(1 / \alpha \rho_{2}, 0\right)$ as initial values and integrating (66), we obtain (7)'s periodic wave solution of implicit function as follows:

$$
\alpha^{2} \rho_{2}^{2} \phi^{2}=\sec \left[\frac{2}{\rho_{2}} \sqrt{-\frac{\rho_{2}}{3 \beta}} \xi-\sqrt{2} F\left(\arccos \left(\frac{1}{\alpha \rho_{2} \phi}\right), \frac{\sqrt{2}}{2}\right)\right] .
$$

(ii) Especially, when $\rho_{2}>0$ and $\rho_{4}=10 \rho_{2}^{2} / 3$, (61) can be reduced to

$$
\begin{aligned}
& \frac{1}{2} \frac{d \phi^{2}}{\phi^{2} \sqrt{\left(1 / \alpha^{2} \rho_{2}^{2}\right)^{2}-\left(\phi^{2}\right)^{2}}}+\frac{\alpha \rho_{2} d \phi}{\sqrt{\left(\phi^{2}+1 / \alpha^{2} \rho_{2}^{2}\right)\left(1 / \alpha^{2} \rho_{2}^{2}-\phi^{2}\right)}} \\
& =\alpha^{2} \rho_{2} \sqrt{\frac{\rho_{2}}{3 \beta}} d \xi
\end{aligned}
$$

where $1 / \alpha^{2} \rho_{2}^{2}>\phi>0$. Taking the $\left(1 / \alpha \rho_{2}, 0\right)$ as initial values and integrating (68), we obtain (7)'s implicit solitary wave solution as follows:

$$
\alpha^{2} \rho_{2}^{2} \phi^{2}=\operatorname{sech}\left[\sqrt{2} F\left(\arccos \left(\alpha \rho_{2} \phi\right), \frac{\sqrt{2}}{2}\right)-\frac{2}{\rho_{2}} \sqrt{\frac{\rho_{2}}{3 \beta}} \xi\right] .
$$

3.4. The Exact Solutions under the Parametric Conditions of Case 4. Substituting (28) into the expression $d \phi / d \xi=y$ of (13) yields

$$
\begin{aligned}
& \frac{d \phi}{d \xi}= \\
& \pm \frac{\phi \sqrt{-(3 / 5) \alpha^{4} \rho_{4} \rho_{2} \phi^{4}+(1 / 5) \alpha^{3}\left(10 \rho_{2}^{2}-3 \rho_{4}\right) \phi^{3}-2 \alpha \phi}}{\sqrt{6 \beta}\left(1+\alpha \rho_{2} \phi\right)}
\end{aligned}
$$

Taking “+”, (70) can be rewritten as

$$
\begin{aligned}
& \frac{\left(1+\alpha \rho_{2} \phi\right) d \phi}{\phi \sqrt{-(3 / 5) \alpha^{4} \rho_{4} \rho_{2} \phi^{4}+(1 / 5) \alpha^{3}\left(10 \rho_{2}^{2}-3 \rho_{4}\right) \phi^{3}-2 \alpha \phi}} \\
& =\frac{1}{\sqrt{6 \beta}} d \xi .
\end{aligned}
$$

(i) When $\rho_{4} \rho_{2}<0$, (71) can be reduced to

$$
\begin{aligned}
& \frac{\left(1+\alpha \rho_{2} \phi\right) d \phi}{\phi \sqrt{\phi^{4}-\left(\left(10 \rho_{2}^{2}-3 \rho_{4}\right) / 3 \alpha \rho_{4} \rho_{2}\right) \phi^{3}+\left(10 / 3 \alpha^{3} \rho_{4} \rho_{2}\right) \phi}} \\
& =\alpha^{2} \sqrt{-\frac{\rho_{4} \rho_{2}}{10 \beta}} d \xi .
\end{aligned}
$$

Next, we discuss the roots of the following equation:

$$
\phi^{4}-\frac{10 \rho_{2}^{2}-3 \rho_{4}}{3 \alpha \rho_{4} \rho_{2}} \phi^{3}+\frac{10}{3 \alpha^{3} \rho_{4} \rho_{2}} \phi=0 .
$$

(a) When $\rho_{4}<5 \rho_{2}^{2} / 6$, (73) has four real roots as follows:

$$
0, \quad-\frac{1}{\alpha \rho_{2}}, \quad \frac{5 \rho_{2} \pm \sqrt{25 \rho_{2}^{2}-30 \rho_{4}}}{3 \alpha \rho_{4}} .
$$

(b) When $\rho_{4}=5 \rho_{2}^{2} / 6$, (73) has still four real roots as follows:

$$
0, \quad-\frac{1}{\alpha \rho_{2}}, \quad \frac{2}{\alpha \rho_{2}}, \quad \frac{2}{\alpha \rho_{2}},
$$

where $2 / \alpha \rho_{2}$ is a double root.

(c) When $\rho_{4}>5 \rho_{2}^{2} / 6$, (73) has two real roots and two complex roots as follows:

$$
0,-\frac{1}{\alpha \rho_{2}}, \quad \frac{5 \rho_{2} \pm i \sqrt{30 \rho_{4}-25 \rho_{2}^{2}}}{3 \alpha \rho_{4}} .
$$

Thus, by using the above parametric conditions, we can obtain three kinds of exact solutions of implicit function type; see the following discussions.

(1) Under the conditions $0<\rho_{4}<5 \rho_{2}^{2} / 6$ and $\rho_{2}<0$, (72) can be reduced to

$$
\begin{aligned}
& \frac{d \phi}{\phi \sqrt{\left(\phi-a_{1}\right)(\phi-0)\left(\phi-c_{1}\right)\left(\phi-d_{1}\right)}} \\
& +\frac{\alpha \rho_{2} d \phi}{\sqrt{\left(\phi-a_{1}\right)(\phi-0)\left(\phi-c_{1}\right)\left(\phi-d_{1}\right)}} \\
& =\alpha^{2} \sqrt{-\frac{\rho_{4} \rho_{2}}{10 \beta}} d \xi,
\end{aligned}
$$

where $a_{1}=-1 / \alpha \rho_{2}, c_{1}=\left(5 \rho_{2}+\sqrt{25 \rho_{2}^{2}-30 \rho_{4}}\right) / 3 \alpha \rho_{4}, d_{1}=$ $\left(5 \rho_{2}-\sqrt{25 \rho_{2}^{2}-30 \rho_{4}}\right) / 3 \alpha \rho_{4}$, and $\phi>a_{1}>0>c_{1}>d_{1}$. Taking $\left(a_{1}, 0\right)$ as initial values and integrating (77), we obtain (7)'s periodic wave solution of implicit function type as follows:

$$
\begin{aligned}
\frac{g}{a_{1} k^{2}} & {\left[\left(k^{2}-\mu^{2}\right) F(\varphi, k)+\mu^{2} E(\varphi, k)\right]+g \alpha \rho_{2} F(\varphi, k) } \\
& =\alpha^{2} \sqrt{-\frac{\rho_{4} \rho_{2}}{10 \beta}} \xi,
\end{aligned}
$$


where $\varphi=\arcsin \sqrt{d_{1}\left(a_{1}-\phi\right) /\left(a_{1}-d_{1}\right) \phi}, \mu=\sqrt{1-a_{1} / d_{1}}$, $g=2 / \sqrt{d_{1}\left(c_{1}-a_{1}\right)}, k=\sqrt{c_{1}\left(d_{1}-a_{1}\right) / d_{1}\left(c_{1}-a_{1}\right)}$.

(2) Under the conditions $\rho_{4}=5 \rho_{2}^{2} / 6$ and $\rho_{2}<0$, (72) can be reduced to

$$
\frac{\left(1+\alpha \rho_{2} \phi\right) d \phi}{\phi\left(\phi-2 / \alpha \rho_{2}\right) \sqrt{\phi\left(\phi+1 / \alpha \rho_{2}\right)}}=-\alpha^{2} \rho_{2} \sqrt{\frac{-\rho_{2}}{12 \beta}} d \xi .
$$

Equation (79) can be rewritten as

$$
\begin{gathered}
\frac{-\alpha \rho_{2} d \phi}{2 \phi \sqrt{\phi\left(\phi+1 / \alpha \rho_{2}\right)}}+\frac{3 \alpha \rho_{2} d \phi}{2\left(\phi-2 / \alpha \rho_{2}\right) \sqrt{\phi\left(\phi+1 / \alpha \rho_{2}\right)}} \\
=-\alpha^{2} \rho_{2} \sqrt{\frac{-\rho_{2}}{12 \beta}} d \xi .
\end{gathered}
$$

Taking $\left(-1 / \alpha \rho_{2}, 0\right)$ as initial values and integrating (80), we obtain (7)'s solitary wave solution of implicit function type as follows:

$$
\begin{aligned}
& \rho_{2} \sqrt{\frac{\phi+1 / \alpha \rho_{2}}{\phi}}-\frac{3 \rho_{2}}{2 \sqrt{6}} \\
& \quad \times\left[\ln \left|\frac{2 / \alpha \rho_{2}+5 \phi+2 \sqrt{6 \phi\left(\phi+1 / \alpha \rho_{2}\right)}}{\alpha \rho_{2} \phi-2}\right|+\ln \left|\alpha \rho_{2}\right|\right] \\
& =-\sqrt{\frac{-\rho_{2}}{12 \beta}} \xi .
\end{aligned}
$$

(3) Under the conditions $\rho_{4}>5 \rho_{2}^{2} / 6$ and $\rho_{2}<0$, (72) can be reduced to

$$
\begin{aligned}
& \frac{d \phi}{\phi \sqrt{\left(\phi-a_{1}\right)(\phi-0)\left(\phi-c_{1}\right)\left(\phi-\bar{c}_{1}\right)}} \\
& +\frac{\alpha \rho_{2} d \phi}{\sqrt{\left(\phi-a_{1}\right)(\phi-0)\left(\phi-c_{1}\right)\left(\phi-\bar{c}_{1}\right)}} \\
& =\alpha^{2} \sqrt{-\frac{\rho_{4} \rho_{2}}{10 \beta}} d \xi,
\end{aligned}
$$

where $a_{1}=-1 / \alpha \rho_{2}, \quad c_{1}=\left(5 \rho_{2}+i \sqrt{30 \rho_{4}-25 \rho_{2}^{2}}\right) / 3 \alpha \rho_{4}, \bar{c}_{1}=$ $\left(5 \rho_{2}-i \sqrt{30 \rho_{4}-25 \rho_{2}^{2}}\right) / 3 \alpha \rho_{4}$, and $0<a_{1}<\phi<+\infty$. Taking $\left(a_{1}, 0\right)$ as initial values and integrating (82), we obtain (7)'s periodic wave solution of implicit function type as follows:

$$
\begin{aligned}
& \frac{(\widehat{B}-\widehat{A}) \widehat{g}}{\widehat{B} a_{1}} \\
& \quad \times\left[F(\varphi, \widehat{k})+\left(1-\alpha_{2}\right) E(\varphi, \widehat{k})+\frac{\left(1-\alpha_{2}\right) \varphi \sqrt{1-\widehat{k}^{2} \varphi}}{1+\sqrt{1-\varphi}}\right] \\
& \quad+g \alpha \rho_{2} F(\varphi, \widehat{k})=\alpha^{2} \sqrt{-\frac{\rho_{4} \rho_{2}}{10 \beta}} \xi,
\end{aligned}
$$

where $\varphi=\arccos \left[\left((\widehat{A}-\widehat{B}) \phi+a_{1} \widehat{B}\right) /\left((\widehat{A}+\widehat{B}) \phi-a_{1} \widehat{B}\right)\right]$, $\widehat{A}=\sqrt{\left(\rho_{4}+5 \rho_{2}^{2}\right)^{2} / \alpha^{2} \rho_{2}^{2} \rho_{4}^{2}+\left(30 \rho_{4}-25 \rho_{2}^{2}\right) / 6 \alpha \rho_{4}}, \widehat{B}=$ $\sqrt{25 \rho_{2}^{2} / 9 \alpha^{2} \rho_{4}^{2}+\left(30 \rho_{4}-25 \rho_{2}^{2}\right) / 6 \alpha \rho_{4}}, \hat{g}=1 / \sqrt{\widehat{A} \widehat{B}}, \widehat{k}=$ $\sqrt{\left((\widehat{A}+\widehat{B})^{2}-a_{1}^{2}\right) / 4 \widehat{A B}}$.

(ii) When $\rho_{4} \rho_{2}>0$, (72) can be rewritten as

$$
\begin{gathered}
\frac{\left(1+\alpha \rho_{2} \phi\right) d \phi}{\phi \sqrt{-\phi^{4}+\left(\left(10 \rho_{2}^{2}+3 \rho_{4}\right) / 3 \alpha \rho_{4} \rho_{2}\right) \phi^{3}-\left(10 / 3 \alpha^{3} \rho_{4} \rho_{2}\right) \phi}} \\
=\alpha^{2} \sqrt{\frac{\rho_{4} \rho_{2}}{10 \beta}} d \xi .
\end{gathered}
$$

(1) Under the conditions $0<\rho_{4}<5 \rho_{2}^{2} / 6$ and $\rho_{2}>0$, (84) can be reduced to

$$
\begin{aligned}
& \frac{d \phi}{\phi \sqrt{\left(a_{2}-\phi\right)\left(\phi-b_{2}\right)(\phi-0)\left(\phi-d_{2}\right)}} \\
& +\frac{\alpha \rho_{2} d \phi}{\sqrt{\left(a_{2}-\phi\right)\left(\phi-b_{2}\right)(\phi-0)\left(\phi-d_{2}\right)}} \\
& =\alpha^{2} \sqrt{\frac{\rho_{4} \rho_{2}}{10 \beta}} d \xi,
\end{aligned}
$$

where $a_{2}=\left(5 \rho_{2}+\sqrt{25 \rho_{2}^{2}-30 \rho_{4}}\right) / 3 \alpha \rho_{4}, b_{2}=\left(5 \rho_{2}-\right.$ $\left.\sqrt{25 \rho_{2}^{2}-30 \rho_{4}}\right) / 3 \alpha \rho_{4}, d_{2}=-1 / \alpha \rho_{2}$, and $a_{2}>\phi>b_{2}>$ $0>d_{2}$. Taking $\left(b_{2}, 0\right)$ as initial values and integrating $(85)$, we obtain (7)'s periodic wave solution of implicit function type as follows:

$$
\begin{gathered}
\frac{g_{2}}{b_{2}}\left[\frac{b_{2}}{d_{2}} F\left(\varphi, k_{2}\right)+\left(1-\frac{b_{2}}{d_{2}}\right) E\left(\varphi, k_{2}\right)\right]+g_{2} \alpha \rho_{2} F\left(\varphi, k_{2}\right) \\
\quad=\alpha^{2} \sqrt{\frac{\rho_{4} \rho_{2}}{10 \beta}} \xi,
\end{gathered}
$$

where $\varphi=\arcsin \sqrt{a_{2}\left(\phi-b_{2}\right) /\left(a_{2}-b_{2}\right) \phi}, g_{2}=2 / \sqrt{a_{2}\left(b_{2}-d_{2}\right)}$, $k_{2}=\sqrt{-d_{2}\left(a_{2}-b_{2}\right) / a_{2}\left(b_{2}-d_{2}\right)}$.

(2) Under the conditions $\rho_{4}=5 \rho_{2}^{2} / 6$ and $\rho_{2}>0,(84) \mathrm{can}$ be reduced to

$$
\begin{aligned}
& \frac{-\alpha \rho_{2} d \phi}{2 \phi \sqrt{-\phi\left(\phi+1 / \alpha \rho_{2}\right)}}+\frac{3 \alpha \rho_{2} d \phi}{2\left(\phi-2 / \alpha \rho_{2}\right) \sqrt{-\phi\left(\phi+1 / \alpha \rho_{2}\right)}} \\
& =\alpha^{2} \rho_{2} \sqrt{\frac{\rho_{2}}{12 \beta}} d \xi .
\end{aligned}
$$


Respectively, taking $\left(-1 / \alpha \rho_{2}, 0\right)$ and $(0,0)$ as initial values, integrating (87), we obtain (7)'s periodic wave solution of implicit function type as follows:

$$
\frac{2+5 \alpha \rho_{2} \phi}{2-\rho_{2} \phi}=\cos \left[\frac{\alpha \rho_{2} \sqrt{-6 \phi\left(\phi+1 / \alpha \rho_{2}\right)}}{\phi}+\alpha \sqrt{\frac{\rho_{2}}{2 \beta}} \xi\right] .
$$

(3) Under the conditions $\rho_{4}>5 \rho_{2}^{2} / 6$ and $\rho_{2}>0$, (84) can be reduced to

$$
\begin{aligned}
& \frac{d \phi}{\phi \sqrt{(0-\phi)\left(\phi-b_{1}\right)\left(\phi-c_{1}\right)\left(\phi-\bar{c}_{1}\right)}} \\
& +\frac{\alpha \rho_{2} d \phi}{\sqrt{(0-\phi)\left(\phi-b_{1}\right)\left(\phi-c_{1}\right)\left(\phi-\bar{c}_{1}\right)}} \\
& =\alpha^{2} \sqrt{\frac{\rho_{4} \rho_{2}}{10 \beta}} d \xi,
\end{aligned}
$$

where $b_{1}=-1 / \alpha \rho_{2}, \quad c_{1}=\left(5 \rho_{2}+i \sqrt{30 \rho_{4}-25 \rho_{2}^{2}}\right) / 3 \alpha \rho_{4}, \bar{c}_{1}=$ $\left(5 \rho_{2}-i \sqrt{30 \rho_{4}-25 \rho_{2}^{2}}\right) / 3 \alpha \rho_{4}$, and $a_{1}<\phi<0$. Taking $\left(a_{1}, 0\right)$ as initial values and integrating (89), we obtain (7)'s periodic wave solution of implicit function type as follows:

$$
\begin{aligned}
& \frac{(\widetilde{A}+\widetilde{B})}{b_{1} \widetilde{A}}\left[\mu_{2} F(\widetilde{\varphi}, m)+\frac{\widetilde{\mu}-\mu_{2}}{1-\widetilde{\mu}^{2}}\left(\Pi\left(\widetilde{\varphi}, \frac{\tilde{\mu}^{2}}{\widetilde{\mu}^{2}-1}, m\right)-\widetilde{\mu} f_{2}\right)\right] \\
& +\alpha \rho_{2} g F(\widetilde{\varphi}, m)=\alpha^{2} \sqrt{\frac{\rho_{4} \rho_{2}}{10 \beta}} \xi,
\end{aligned}
$$

where constants $\widetilde{A}, \widetilde{B}, \widetilde{g}$ are given above, the $\widetilde{\varphi}=\arccos [(\widetilde{B}(\nu-$ $\phi)-\widetilde{A}(\phi-\gamma)) /(\widetilde{B}(\nu-\phi)+\widetilde{A}(\phi-\gamma))], m^{2}=\left((\nu-\gamma)^{2}-(\widetilde{A}-\right.$ $\left.\widetilde{B})^{2}\right) / 4 \widetilde{A} \widetilde{B}, \widetilde{\mu}=(\gamma \widetilde{A}-v \widetilde{B}) /(\gamma \widetilde{A}+\nu \widetilde{B}), \mu_{2}=(\widetilde{A}-\widetilde{B}) /(\widetilde{A}+\widetilde{B})$, and

$$
\begin{aligned}
f_{2} & =\sqrt{\frac{1-\widetilde{\mu}^{2}}{m^{2}+m^{\prime 2} \widetilde{\mu}^{2}}} \arctan \left[\tilde{\varphi} \sqrt{\frac{m^{2}+m^{\prime 2} \widetilde{\mu}^{2}}{\left(1-\widetilde{\mu}^{2}\right)\left(1-m^{2} \widetilde{\varphi}^{2}\right)}}\right], \\
& \quad \text { if } \frac{\widetilde{\mu}^{2}}{\widetilde{\mu}^{2}-1}<m^{2} ; \\
& \frac{\widetilde{\varphi}}{\sqrt{1-m^{2} \widetilde{\varphi}^{2}}}, \quad \text { if } \frac{\widetilde{\mu}^{2}}{\widetilde{\mu}^{2}-1}=m^{2} ;
\end{aligned}
$$

$$
\begin{gathered}
=\frac{1}{2} \sqrt{\frac{\widetilde{\mu}^{2}-1}{m^{2}+m^{\prime 2} \widetilde{\mu}^{2}}} \\
\times \ln \left[\frac{\sqrt{\left(m^{2}+m^{\prime 2} \widetilde{\mu}^{2}\right)\left(1-m^{2} \widetilde{\varphi}^{2}\right)}+\widetilde{\varphi} \sqrt{\widetilde{\mu}^{2}-1}}{\sqrt{\left(m^{2}+m^{\prime 2} \widetilde{\mu}^{2}\right)\left(1-m^{2} \widetilde{\varphi}^{2}\right)}-\widetilde{\varphi} \sqrt{\widetilde{\mu}^{2}-1}}\right], \\
\text { if } \frac{\widetilde{\mu}^{2}}{\widetilde{\mu}^{2}-1}>m^{2} .
\end{gathered}
$$

3.5. The Exact Solutions under the Parametric Conditions of Case 5. Under different parameters, (29) can be reduced different types. As an example, here we only discuss one reduced to type under the parametric conditions $\rho_{1}>0, \rho_{2}>0$, $\rho_{4}<0$ for (29); the others can be similarly discussed.

Especially, when $\rho_{1}>0, \rho_{2}>0$ and $\rho_{4}<0,(29)$ can be reduced to

$$
\begin{aligned}
y= & \pm \alpha^{2} \sqrt{-\frac{\rho_{2} \rho_{4}}{10 \beta}} \\
& \times\left(\phi \sqrt{\left[\phi^{3}+\frac{5 \rho_{1}}{3 \alpha \rho_{4}} \phi^{2}+\frac{10}{3 \alpha^{2} \rho_{4}} \phi-\frac{10(c-1)}{\alpha^{3} \rho_{4}}\right]\left[\phi-\left(\frac{1}{\alpha \rho_{2}}\right)\right]}\right) \\
& \times\left(1+\alpha \rho_{2} \phi\right)^{-1} .
\end{aligned}
$$

Also, we only consider one case where the equation $\phi^{3}+$ $\left(5 \rho_{1} / 3 \alpha \rho_{4}\right) \phi^{2}+\left(10 / 3 \alpha^{2} \rho_{4}\right) \phi-\left(10(c-1) / \alpha^{3} \rho_{4}\right)=0$ has three real roots here. The other two cases (i.e., two real roots and one real root) can be similarly discussed. Because the expressions of these three roots are very complex, we use the symbols $\phi_{1}, \phi_{2}, \phi_{3}$ instead of them. In fact, once the parameters are fixed, these three roots can be solved by the above equation. For example, if $\alpha=0.5, \beta=0.6, c=2, \rho_{1}=$ $6, \rho_{4}=-2$, then $\phi_{1} \doteq 1.843, \phi_{2} \doteq 10.270, \phi_{3} \doteq-2.113$. In addition, if $\rho_{2}=5$, then $-1 / \alpha \rho_{2}=-0.4>\phi_{3}$; if $\rho_{2}=1 / 5$, then $-1 / \alpha \rho_{2}=-10<\phi_{3}$. Thus, under the above parametric conditions, (92) can be rewritten as

$$
\begin{array}{r}
y= \pm \alpha^{2} \sqrt{-\frac{\rho_{2} \rho_{4}}{10 \beta}} \frac{\phi \sqrt{\left(\phi-\phi_{1}\right)\left(\phi-\phi_{2}\right)\left(\phi-\phi_{3}\right)(\phi-\delta)}}{1+\alpha \rho_{2} \phi} \\
\left(\phi_{1}>\phi_{2}>\delta>\phi_{3}\right),
\end{array}
$$

or

$$
\begin{aligned}
& y= \pm \alpha^{2} \sqrt{-\frac{\rho_{2} \rho_{4}}{10 \beta}} \frac{\phi \sqrt{\left(\phi-\phi_{1}\right)\left(\phi-\phi_{2}\right)(\phi-\delta)\left(\phi-\phi_{3}\right)}}{1+\alpha \rho_{2} \phi}, \\
& \left(\phi_{3}<\delta<\phi_{2}<\phi_{1}\right) \text {, }
\end{aligned}
$$

where $\delta=-1 / \alpha \rho_{2}$. 


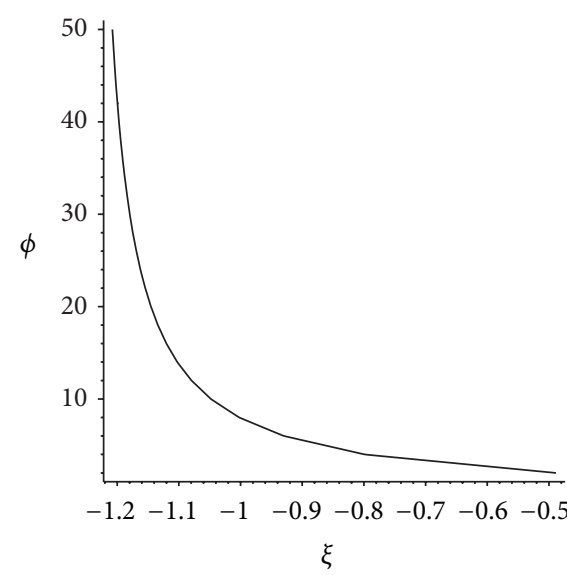

(a) Bounded wave

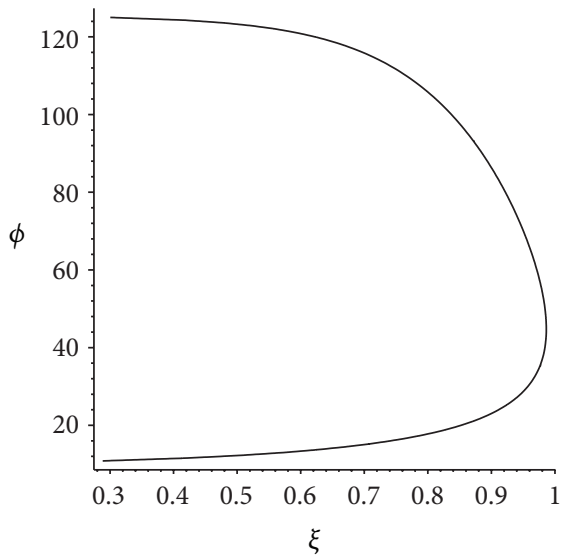

(c) Broken wave of U-form

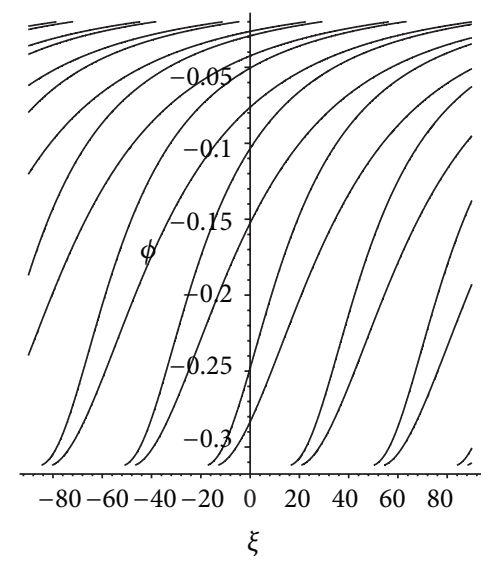

(b) Periodic wave of singular type

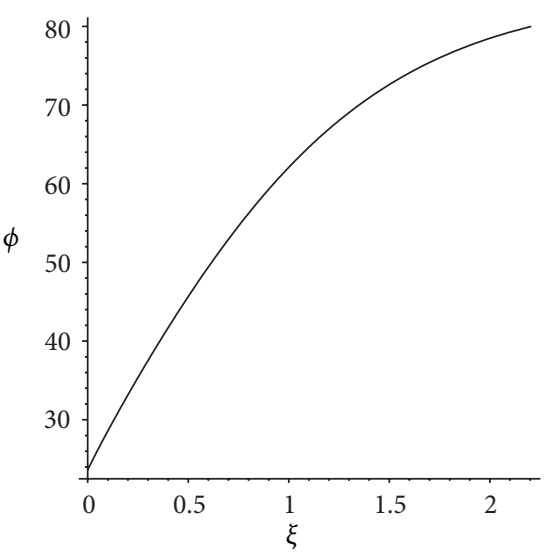

(d) Broken wave of semiparabola form

FIgURE 3: The profiles of solutions (81), (88), (96), and (97) for the fixed parameters.

Respectively, substituting (93) and (94) into the equation $d \phi / d \xi=y$ of (13) yields

$$
\begin{aligned}
& \frac{\left(1+\alpha \rho_{2} \phi\right) d \phi}{\phi \sqrt{\left(\phi-\phi_{1}\right)\left(\phi-\phi_{2}\right)\left(\phi-\phi_{3}\right)(\phi-\delta)}}= \pm \alpha^{2} \sqrt{-\frac{\rho_{2} \rho_{4}}{10 \beta}} d \xi, \\
& \frac{\left(1+\alpha \rho_{2} \phi\right) d \phi}{\phi \sqrt{\left(\phi-\phi_{1}\right)\left(\phi-\phi_{2}\right)(\phi-\delta)\left(\phi-\phi_{3}\right)}}= \pm \alpha^{2} \sqrt{-\frac{\rho_{2} \rho_{4}}{10 \beta}} d \xi .
\end{aligned}
$$

Taking $\left(\phi_{1}, 0\right)$ as initial values and integrating $(95)$, respectively, we obtain (7)'s two groups of exact solutions of implicit function type as follows:

$$
\begin{gathered}
\frac{g_{1}}{\alpha_{1}^{2} \phi_{2}}\left[\left(\frac{\alpha_{1}^{2} \phi_{2}}{\phi_{1}}-\alpha_{1}^{2}\right) \Pi\left(\vartheta_{1}, \alpha_{1}^{2}, \kappa_{1}\right)+\alpha_{1}^{2} F\left(\vartheta_{1}, \kappa_{1}\right)\right] \\
+g_{1} \alpha \rho_{2} F\left(\vartheta_{1}, \kappa_{1}\right)= \pm \alpha^{2} \sqrt{-\frac{\rho_{2} \rho_{4}}{10 \beta}} \xi
\end{gathered}
$$

$$
\begin{aligned}
\frac{g_{2}}{\alpha_{2}^{2} \phi_{2}} & {\left[\left(\frac{\alpha_{2}^{2} \phi_{2}}{\phi_{1}}-\alpha_{2}^{2}\right) \Pi\left(\vartheta_{2}, \alpha_{2}^{2}, \kappa_{2}\right)+\alpha_{2}^{2} F\left(\vartheta_{2}, \kappa_{2}\right)\right] } \\
+ & g_{2} \alpha \rho_{2} F\left(\vartheta_{2}, \kappa_{2}\right)= \pm \alpha^{2} \sqrt{-\frac{\rho_{2} \rho_{4}}{10 \beta}} \xi,
\end{aligned}
$$

where $g_{1}=2 / \sqrt{\left(\phi_{1}-\phi_{3}\right)\left(\phi_{2}-\delta\right)}, g_{2}=2 / \sqrt{\left(\phi_{1}-\delta\right)\left(\phi_{2}-\phi_{3}\right)}$, $\alpha_{1}^{2}=\left(\phi_{1}-\delta\right) /\left(\phi_{2}-\delta\right)>1, \alpha_{2}^{2}=\left(\phi_{1}-\phi_{3}\right) /\left(\phi_{2}-\phi_{3}\right)>1$, $\vartheta_{1}=\arcsin \sqrt{\left(\phi_{2}-\delta\right)\left(\phi-\phi_{1}\right) /\left(\phi_{1}-\delta\right)\left(\phi-\phi_{2}\right)}$, $\vartheta_{2}=\arcsin \sqrt{\left(\phi_{2}-\phi_{3}\right)\left(\phi-\phi_{1}\right) /\left(\phi_{1}-\phi_{3}\right)\left(\phi-\phi_{2}\right)}$, $\kappa_{1}=\sqrt{\left(\phi_{2}-\phi_{3}\right)\left(\phi_{1}-\delta\right) /\left(\phi_{1}-\phi_{3}\right)\left(\phi_{2}-\delta\right)}$, $\kappa_{2}=\sqrt{\left(\phi_{2}-\delta\right)\left(\phi_{1}-\phi_{3}\right) /\left(\phi_{1}-\delta\right)\left(\phi_{2}-\phi_{3}\right)}$.

Among the above traveling wave solutions, it is also worthy to mention solutions (81), (88), (96), and (97); they have some peculiar dynamical properties, too. Solution (81) denotes a bounded wave with level asymptote under the fixed parameters $\alpha=0.7, \beta=0.4, \rho_{2}=-2$. Solution (88) denotes a periodic wave of singular type under the fixed parameters $\alpha=0.8, \beta=0.6, \rho_{2}=4$. Solution (96) denotes a broken wave of $\mathrm{U}$-form (leftward fallen $\mathrm{U}$ ) under the fixed parameters $\alpha=0.5, \beta=0.4, \rho_{2}=5, c=2, \rho_{1}=6, \rho_{4}=-2$, $\phi_{1} \approx 1026989443, \phi_{2} \approx 1.843205997, \phi_{3} \approx-2.113100425$, 
$\delta=-0.40$. Solution (97) denotes a broken wave of semiparabola form under the fixed parameters $\alpha=0.5, \beta=0.4$, $\rho_{2}=0.2, c=2, \rho_{1}=6, \rho_{4}=-2, \phi_{1} \approx 1026989443$, $\phi_{2} \approx 1.843205997, \phi_{3} \approx-2.113100425, \delta=-10$. In order to describe the dynamic properties of the above traveling wave solutions intuitively, similarly, we, respectively, draw profile of solutions (81), (88), (96), and (97) by using software Maple, which are shown in Figure 3.

\section{Conclusion}

In this work, by using the integral bifurcation method, we studied a generalized KdV equation of neglecting the highest order infinitesimal term. Some singular and nonsingular traveling wave solutions including singular solitary wave solutions, semiloop soliton solutions, dark soliton solutions, dark peakon solutions, dark loop-soliton solutions, broken wave solutions of $\mathrm{C}$-form and $\mathrm{U}$-form, periodic wave solutions of singular type, and broken wave solution of semiparabola form were obtained. Among these traveling wave solutions, the solutions such as (40), (46), (47), (81), (88), (96), and (97) have some peculiar dynamical properties and very interesting traveling wave phenomena. It is very worthy to mention solution (47); it denotes a dark peakon, a dark loop soliton, a broken loop soliton, a bright soliton, and a singular compacton when the parameter $\rho_{1}$ varies. This course of changes of waveform shows that solution (47) is a continuous solution. Indeed, the loop soliton which contains (47) is also continuous; this case has proved again that the loop soliton solution of some nonlinear traveling wave equation is one continuous solution, not three breaking solutions; see the arguments in $[23,24]$. However, the phenomenon of half loop soliton exists in troth. For example, the semiloop soliton and broken loop soliton show that their waveforms are only a part of a whole loop soliton. Maybe, a loop soliton can be divided into two parts or three parts like the semiloop soliton or three breaking waves which appeared in [24], but every part of them is still continuous, and then joining them together, they come into being a continuous loop soliton again. Indeed, in 2006, Vakhnenko and Parkes's work [25] successfully explained similar phenomena. In [25], the graphical interpretation of the solution for gDPE is presented. In this analysis, the 3D-spiral (whether one loop from a spiral or a half loop of a spiral) has the different projections that is the essence of the possible solutions.

The results obtained in this work are very different from those in other literatures because this special case of (1) for $\rho_{5}=\rho_{6}=\rho_{7}=0, \rho_{3}=2 \rho_{2}$ is not studied in other references. Especially, the results of (7) are different from those of (4) because of $\rho_{4} \neq 0$. In addition, we hope more and more researchers pay attention to investigations of exact solutions for (7) under general conditions in the future work.

\section{Acknowledgments}

The authors thank reviewers very much for their useful comments and helpful suggestions. This work was financially supported by the Natural Science Foundation of China
(Grant no. 11161038). It was also supported by the Natural Science Foundations of Zhejiang Province (Grant no. Y2111160) and Yunnan Province (Grant no. 2011FZ193).

\section{References}

[1] A. S. Fokas, "On a class of physically important integrable equations," Physica D. Nonlinear Phenomena, vol. 87, no. 1-4, pp. 145-150, 1995.

[2] E. Tzirtzilakis, V. Marinakis, C. Apokis, and T. Bountis, "Soliton-like solutions of higher order wave equations of the Korteweg-de Vries type," Journal of Mathematical Physics, vol. 43, no. 12, pp. 6151-6165, 2002.

[3] E. Tzirtzilakis, M. Xenos, V. Marinakis, and T. C. Bountis, "Interactions and stability of solitary waves in shallow water," Chaos, Solitons \& Fractals, vol. 14, no. 1, pp. 87-95, 2002.

[4] J. Li, W. Rui, Y. Long, and B. He, "Travelling wave solutions for higher-order wave equations of KdV type. III," Mathematical Biosciences and Engineering, vol. 3, no. 1, pp. 125-135, 2006.

[5] W. Rui, Y. Long, and B. He, "Some new travelling wave solutions with singular or nonsingular character for the higher order wave equation of KdV type (III)," Nonlinear Analysis: Theory, Methods \& Applications, vol. 70, no. 11, pp. 3816-3828, 2009.

[6] B. Fuchssteiner and A. S. Fokas, "Symplectic structures, their Bäcklund transformations and hereditary symmetries," Physica D. Nonlinear Phenomena, vol. 4, no. 1, pp. 47-66, 1981.

[7] R. Camassa and D. D. Holm, "An integrable shallow water equation with peaked solitons," Physical Review Letters, vol. 71, no. 11, pp. 1661-1664, 1993.

[8] Q. Bi, "Peaked singular wave solutions associated with singular curves," Chaos, Solitons \& Fractals, vol. 31, no. 2, pp. 417-423, 2007.

[9] J. Li, J. Wu, and H. Zhu, "Traveling waves for an integrable higher order KdV type wave equations," International Journal of Bifurcation and Chaos in Applied Sciences and Engineering, vol. 16, no. 8, pp. 2235-2260, 2006.

[10] J. Li, "Dynamical understanding of loop soliton solution for several nonlinear wave equations," Science in China. Series A. Mathematics, vol. 50, no. 6, pp. 773-785, 2007.

[11] V. Marinakis, "Integrable third order equations of the KdV type," Journal of Mathematical Sciences. Advances and Applications, vol. 5, no. 2, pp. 317-332, 2010.

[12] V. Marinakis, "Higher-order equations of the KdV type are integrable," Advances in Mathematical Physics, vol. 2010, Article ID 329586, 5 pages, 2010.

[13] M. Gandarias and M. S. Bruzon, "Conservation laws for a third order equation by using freesoftware MAXIMA," in Recent Researches in Engineering Education and Software Engineering, pp. 48-51, WSEAS Press, 2012.

[14] J. Li and J. Zhang, "Bifurcations of travelling wave solutions for the generalization form of the modified KdV equation," Chaos, Solitons \& Fractals, vol. 21, no. 4, pp. 889-913, 2004.

[15] S. A. Khuri, "Soliton and periodic solutions for higher order wave equations of KdV type. I," Chaos, Solitons \& Fractals, vol. 26, no. 1, pp. 25-32, 2005.

[16] Y. Long, W. Rui, and B. He, "Travelling wave solutions for a higher order wave equations of KdV type. I," Chaos, Solitons and Fractals, vol. 23, no. 2, pp. 469-475, 2005.

[17] R. Kumar, K. Chong, and J. Park, "Exact null controllability of KdV-burgers equation with memory effect systems," Abstract and Applied Analysis, vol. 2012, Article ID 531659, 20 pages, 2012. 
[18] W. Zhang and X. Li, "Approximate damped oscillatory solutions for generalized KdV-Burgers equation and their error estimates," Abstract and Applied Analysis, vol. 2011, Article ID 807860, 26 pages, 2011.

[19] J.-M. Kim and C. Chun, "New exact solutions to the KdVBurgers-Kuramoto equation with the Exp-function method," Abstract and Applied Analysis, vol. 2012, Article ID 892420, 10 pages, 2012.

[20] W. Rui, B. He, Y. Long, and C. Chen, "The integral bifurcation method and its application for solving a family of thirdorder dispersive PDEs," Nonlinear Analysis: Theory, Methods \& Applications, vol. 69, no. 4, pp. 1256-1267, 2008.

[21] R. Weiguo, L. Yao, H. Bin, and L. Zhenyang, "Integral bifurcation method combined with computer for solving a higher order wave equation of KdV type," International Journal of Computer Mathematics, vol. 87, no. 1-3, pp. 119-128, 2010.

[22] V. O. Vakhnenko and E. J. Parkes, "Periodic and solitary-wave solutions of the Degasperis-Procesi equation," Chaos, Solitons \& Fractals, vol. 20, no. 5, pp. 1059-1073, 2004.

[23] K. K. Victor, B. B. Thomas, and C. K. Timoleon, "Reply to "comment on "on two-loop soliton solution of the Schäfer-Wayne short-pulse equation using Hirota's method and HodnettMoloney approach"',', Journal of the Physical Society of Japan, vol. 76, no. 11, Article ID 116002, pp. 116002-1-116002-2, 2007.

[24] Y. Zhang and J. Li, "Comment on "on two-loop soliton solution of the Schäfer-Wayne shortpulse equation using hirota's method and hodnett-molony approach," Journal of the Physical Society of Japan, vol. 76, no. 11, pp. 116001-1-116001-2, 2007.

[25] V. O. Vakhnenko and E. J. Parkes, “The solutions of a generalized Degasperis-Procesi equation," Reports of the National Academy of Sciences of Ukraine, no. 8, pp. 88-94, 2006. 


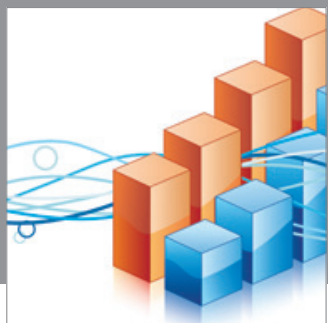

Advances in

Operations Research

mansans

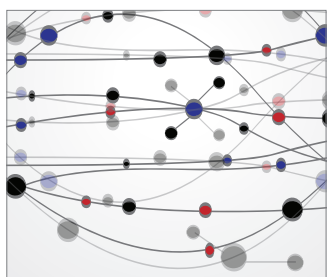

The Scientific World Journal
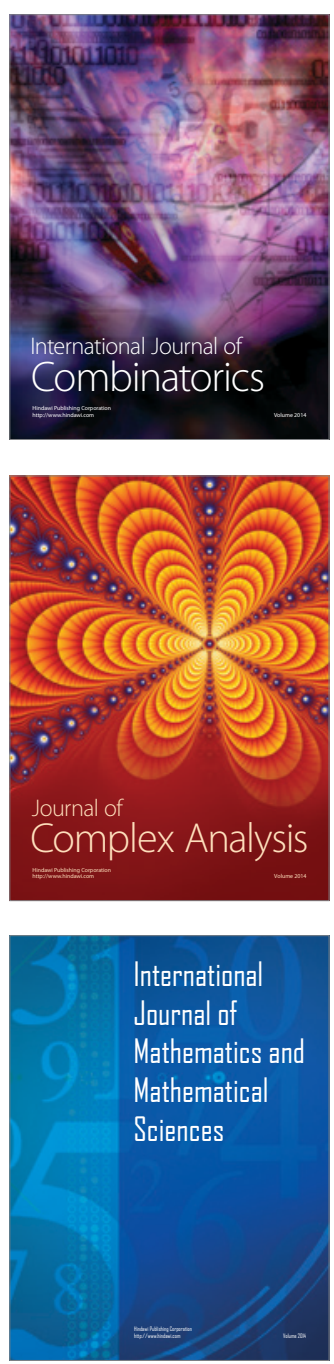
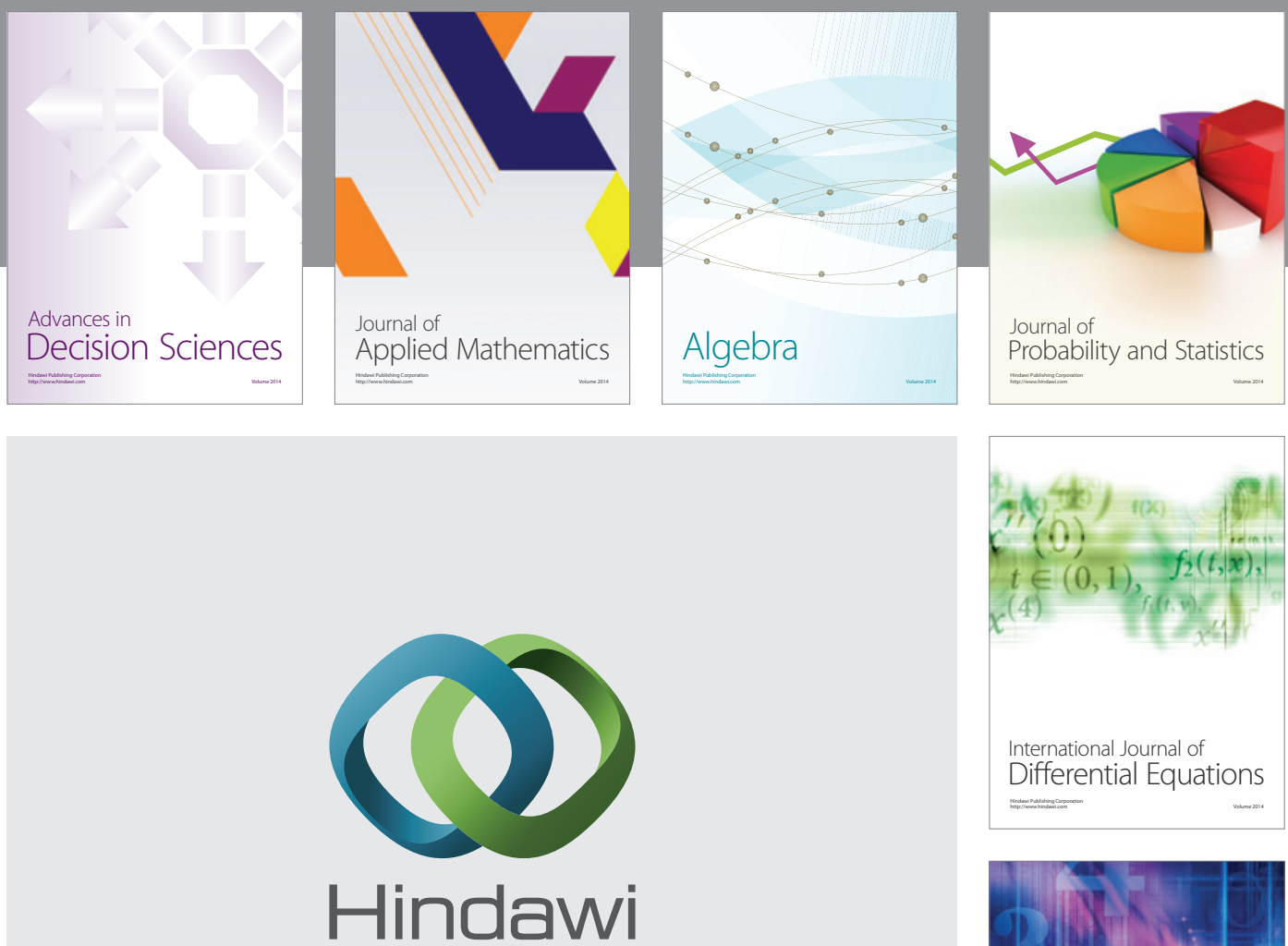

Submit your manuscripts at http://www.hindawi.com
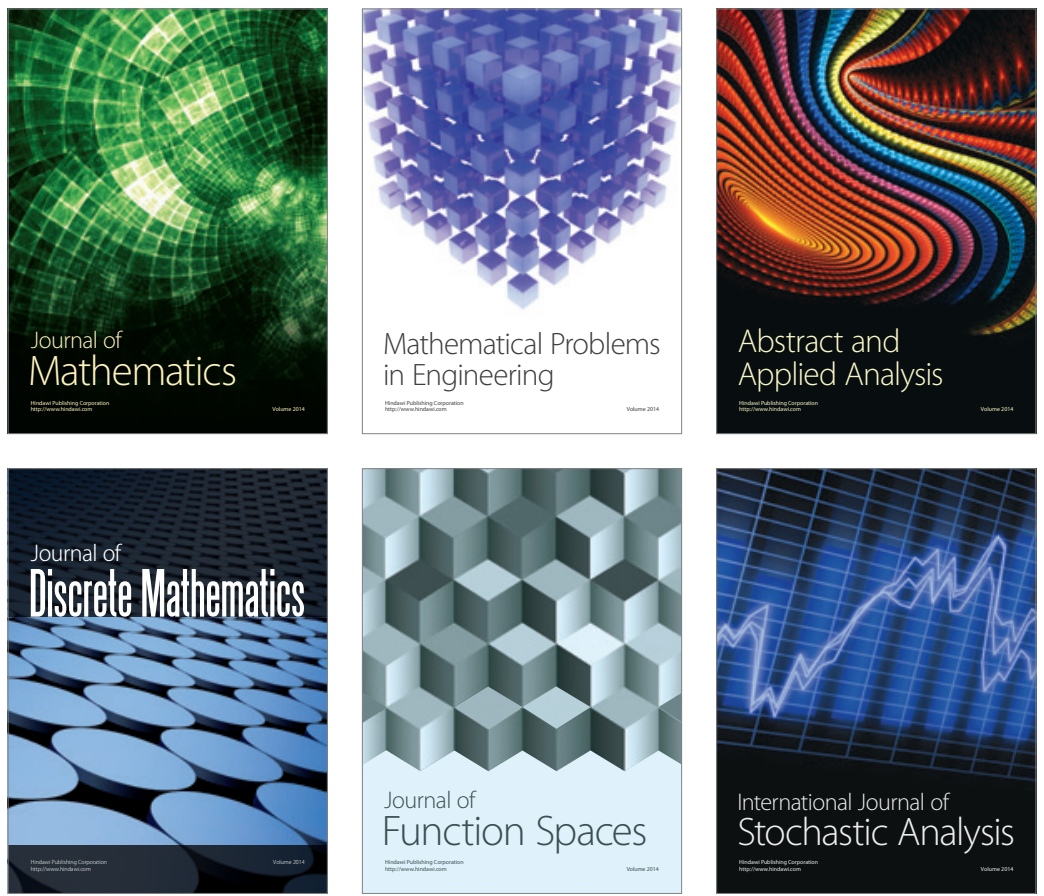

Journal of

Function Spaces

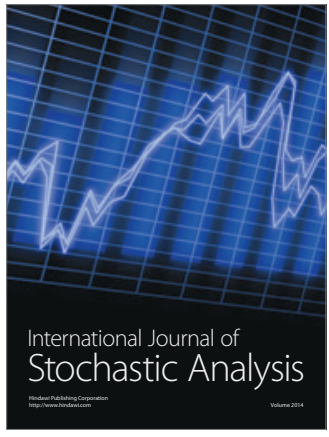

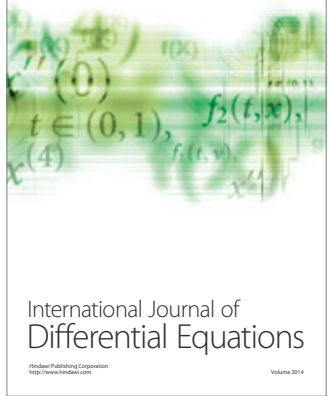
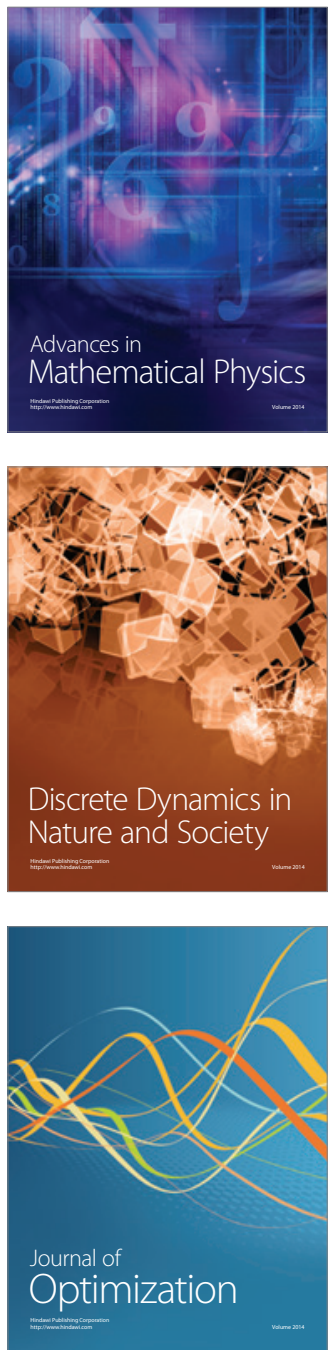